\title{
Edge disjoint Hamiltonian cycles in highly connected tournaments
}

\author{
Alexey Pokrovskiy ${ }^{1}$ \\ ${ }^{1}$ Methods for Discrete Structures, Freie Universität, Berlin, Germany. \\ Correspondence to be sent to: alja123@gmail.com
}

\begin{abstract}
Thomassen conjectured that there is a function $f(k)$ such that every strongly $f(k)$-connected tournament contains $k$ edge-disjoint Hamiltonian cycles. This conjecture was recently proved by Kühn, Lapinskas, Osthus, and Patel who showed that $f(k) \leq O\left(k^{2}(\log k)^{2}\right)$ and conjectured that there is a constant $C$ such that $f(k) \leq C k^{2}$. We prove this conjecture. As a second application of our methods we answer a question of Thomassen about spanning linkages in highly connected tournaments.
\end{abstract}

\section{Introduction}

A directed graph is Hamiltonian if there is a directed cycle passing through all its vertices. Hamiltonicity has a very long history in both directed and undirected graphs, and there are many results guaranteeing that a graph is Hamiltonian under certain conditions (see $[1,8]$ ).

In general, it is hard to decide whether a directed graph is Hamiltonian - the problem is well known to be NP complete, even for undirected graphs. However for the special case of tournaments the problem becomes easier (a tournament is a directed graph which has exactly one edge between any pair of vertices). Here, an old result of Camion [3] says that a tournament is Hamiltonian if, and only if, it is strongly connected i.e. for any two vertices $x$ and $y$ there is a directed path from $x$ to $y$. Since strong-connectedness can be tested in polynomial time, this gives an efficient algorithm for testing whether a tournament is Hamiltonian.

Many results about Hamiltonicity have focused on finding several Hamiltonian cycles. Often one wants to count how many different Hamiltonian cycles there are, or to pack several edge-disjoint Hamiltonian cycles in a graph (see [8]). One natural condition for finding edge-disjoint Hamiltonian cycles in a tournament is strong $k$-connectedness. A directed graph is strongly $k$-connected if it remains strongly connected after the removal of any set of $k-1$ vertices. Thomassen made the following conjecture about finding edge disjoint Hamiltonian cycles in a highly connected tournament.

Conjecture 1.1 (Thomassen, [13]). There is a function $f(k)$ such that every strongly $f(k)$-connected tournament contains $k$ edge-disjoint Hamiltonian cycles.

From Camion's Theorem, we have $f(1)=1$. For all larger $k$, Conjecture 1.1 was proved by Kühn, Lapinskas, Osthus, and Patel.

Theorem 1.2 (Kühn, Lapinskas, Osthus, and Patel, [7]). There is a constant $C$ such that every strongly $C k^{2}(\log k)^{2}$-connected tournament contains $k$ edge-disjoint Hamiltonian cycles.

The $C k^{2}(\log k)^{2}$ bound on the connectedness in the above theorem is close to best possible. Indeed Kühn, Lapinskas, Osthus, and Patel constructed tournaments which are strongly $(k-1)^{2} / 4$-connected, but have no $k$ edge-disjoint Hamiltonian cycles [7]. They conjectured that the $\log k$ factors in Theorem 1.2 were unnecessary and a $C k^{2}$ bound on the connectivity should suffice.

Conjecture 1.3 (Kühn, Lapinskas, Osthus, and Patel, [7]). There is a constant $C$ such that every strongly $C k^{2}$-connected tournament contains $k$ edge-disjoint Hamiltonian Cycles.

The main result of this paper is a proof of this conjecture.

Theorem 1.4. There is a constant $C$ such that every strongly $C k^{2}$-connected tournament contains $k$ edgedisjoint Hamiltonian Cycles. 
This theorem is proved using the method of linkage structures in tournaments. This technique was introduced in [7] during the proof of Theorem 1.2. Since then the technique has found other applications in $[6,9,10]$ to prove results about highly connected tournaments. The following is an informal definition of what a linkage structure is

A linkage structure $L$ in a tournament $T$, is a small subset of $V(T)$ with the property that for many pairs of vertices $x, y$ outside $L$, there is a path from $x$ to $y$ most of whose vertices are contained in $L$.

This definition is purposefully vague in order to include all previously used linkage structures. Since linkage structures arose with specific applications in mind, the exact meaning of "small," "many," and "most" in the above definition varies depending on what application one is looking at. In applications, one first proves an intermediate result which shows that every highly connected tournament contains many disjoint linkage structures. Then these linkage structures are used to build whatever object one is looking for in the tournament (in our case Hamiltonian cycles).

The second result proved in this paper is about spanning linkages in tournaments. For two ordered sets of vertices $X=\left(x_{1}, \ldots, x_{k}\right)$ and $Y=\left(y_{1}, \ldots, y_{k}\right)$ in a tournament $T$, a linkage between $X$ and $Y$ is a collection of vertex disjoint paths $P_{1}, \ldots, P_{k}$ with $P_{i}$ going from $x_{i}$ to $y_{i}$. The linkage is said to be spanning if $V\left(P_{1}\right) \cup \cdots \cup V\left(P_{k}\right)=V(T)$. Thomassen proved that there is a function $g(k)$ such that every strongly $g(k)$ connected tournament has a spanning linkage between any two disjoint ordered sets of $k$ vertices [14]. The function $g(k)$ in Thomassen's proof is $O\left(2^{k} k !\right)$, and Thomassen asked whether a linear function suffices. Kim, Kühn, and Osthus proved a quadratic upper bound on $g(k)$ in [6]. In [10], the author showed that every strongly $452 k$-connected tournament has a (not necessarily spanning) linkage between any two sets of vertices. In this paper we are able to use our linkage structures to answer Thomassen's question.

Theorem 1.5. There is a constant $C$ such that every $C k$-connected tournament $T$ and two sets of vertices $X=\left(x_{1}, \ldots, x_{k}\right)$ and $Y=\left(y_{1}, \ldots, y_{k}\right)$ in $T$, there is a spanning linkage from $X$ to $Y$.

The structure of this paper is as follows. In the next section, we state what properties our linkage structures have, and use them to deduce Theorem 1.4. In Section 3 we prove Theorem 1.5. In Section 4, we define our linkage structures (which we call "linkers") and derive their properties. Finally, in Section 5 we give some concluding remarks and open problems.

\section{Finding Hamiltonian cycles using linkage structures}

In this section we formally introduce linkage structures, state the main theorem about them that we will need, and use it to deduce Theorem 1.4.

A directed graph is Hamiltonian connected if for any pair of vertices $x$ and $y$, it contains a Hamiltonian path from $x$ to $y$. The following is a version of a theorem of Kühn, Osthus, and Townsend. It is perhaps the simplest example of linkage structures to state.

Theorem 2.1 (Kühn, Osthus, and Townsend, [9]). All strongly $10^{16} k^{3} \log \left(k^{2}\right)$-connected tournaments contain disjoint $k$ sets of vertices $L_{1}, \ldots, L_{k}$ such that

- $\left|L_{i}\right| \leq|T| / 100 k$.

- For any $S \subseteq T \backslash\left(L_{1} \cup \cdots \cup L_{k}\right)$, the subtournament on $L_{i} \cup S$ is Hamiltonian connected for every $i$.

This theorem is obtained from combining Theorem 1.5 from [9] with a theorem of Thomassen that every strongly 4-connected tournament is Hamiltonian connected [12].

Comparing this theorem with the informal definition of linkage structures given in the introduction, we see that for any pair of vertices $x, y$ outside of the linkage structures $L_{1}, \ldots, L_{k}$, there is a path from $x$ to $y$, all of whose internal vertices are contained in any one of the linkage structures $L_{i}$.

It is easy to see how Theorem 2.1 might be useful in proving results about Hamiltonicity of tournaments. Indeed suppose that we have sets $L_{1}, \ldots, L_{k}$ as in Theorem 2.1. Then for any partition of $T \backslash\left(L_{1} \cup \cdots \cup L_{k}\right)$ into $k$ paths $P_{1}, \ldots, P_{k}$, there is a Hamiltonian cycle in $T$ containing $P_{1}, \ldots, P_{k}$. Indeed this cycle is obtained by successively considering pairs of paths $P_{i}$ and $P_{i+1}(\bmod k)$. If $x$ is the end of $P_{i}$ and $y$ is the start of $P_{i+1}(\bmod k)$, then Theorem 2.1 implies that there is a Hamiltonian path from $x$ to $y$ in $L_{i}+x+y$. This Hamiltonian path is used to join $P_{i}$ to $P_{i+1}(\bmod k)$ using all the vertices of $L_{i}$. Repeating this for all $i=1, \ldots, k$, produces the required Hamiltonian cycle. 
The following is main idea of the proofs of Theorems 1.2 and 1.4. First we use a result similar to Theorem 2.1 to find many disjoint linkage structures in a highly connected tournament $T$. Then, we find $k$ collections of edgedisjoint paths, each collection partitioning the remaining vertices of $T$. Finally, using the linkage structures we join each collection of paths into a Hamiltonian cycle. A similar strategy was used in [7] to prove Theorem 1.2. The main novelty in our proof is that we are able to find more linkage structures in a tournament of given connectivity, which leads to the improved bound in Theorem 1.4.

To find the collections of paths, we use a theorem of Gallai and Milgram. The independence number of a directed graph is the order of the largest subset of vertices with no edges inside it.

Theorem 2.2 (Gallai-Milgram, [5]). Let $D$ be a directed graph with independence number $k$. Then $V(D)$ can be covered by at most $k$ vertex disjoint paths.

The degree of a vertex in a directed graph is the sum of its in and out-degrees. Notice that a directed graph with minimum degree $n-k-1$ must have independence number at most $k$. Therefore the above theorem has the following corollary.

Corollary 2.3. Let $D$ be a directed graph with minimum degree $\geq n-k-1$. Then $V(D)$ can be covered by at most $k$ vertex disjoint paths.

Repeatedly applying this corollary to a tournament $T$ produces collections of paths $\mathcal{P}_{1}, \ldots, \mathcal{P}_{k}$ such that $\mathcal{P}_{i}$ consists of $2 i-1$ vertex disjoint paths which cover $V(T)$, and also for all $i \neq j$ the paths in $\mathcal{P}_{i}$ are edge-disjoint from those in $\mathcal{P}_{j}$. It is the paths in these collections which the linkage structures join into Hamiltonian cycles. Assuming we need $2 i-1$ linkage structures to join the $2 i-1$ paths in $\mathcal{P}_{i}$ into a cycle, we would need $k^{2}$ linkage structures altogether. This is the source of the quadratic bound in Theorems 1.2 and 1.4.

Next, we formally define the properties of the linkage structures we use. We will actually define a family of several linkage structures which we call a linking family.

Definition 2.4. For $k \geq 1$, a family $\left\{L_{1}, \ldots, L_{k}\right\}$ of vertex disjoint subdigraphs of a digraph $D$ is a linking family of size $k$ in $D$ if the following holds.

Suppose we have two vertices $x$ and $y$ outside $L_{1} \cup \cdots \cup L_{k}$ and at most $100 k$ vertex disjoint paths $P_{1}, \ldots, P_{m}$ in $V(T) \backslash\left(L_{1} \cup \cdots \cup L_{k} \cup\{x, y\}\right)$. Then there are paths $P, P_{1}^{\prime}, \ldots, P_{m}^{\prime}$ and subdigraphs $L_{1}^{\prime}, \ldots, L_{k-1}^{\prime}$ all of which are vertex disjoint, such that

(i) $P$ is from $x$ to $y$.

(ii) $P \cup P_{1}^{\prime} \cup \cdots \cup P_{m}^{\prime} \cup L_{1}^{\prime} \cup \cdots \cup L_{k-1}^{\prime}$ consists of $L_{1} \cup \cdots \cup L_{k} \cup P_{1} \cup \cdots \cup P_{m} \cup\{x, y\}$, plus at most 6 other vertices.

(iii) $P_{j}^{\prime}$ has the same endpoints as $P_{j}$ for every $j$.

(iv) If $k \geq 2$, then $\left\{L_{1}^{\prime}, \ldots, L_{k-1}^{\prime}\right\}$ is a linking family of size $k-1$ in $D$.

Part (iv) of this definition may look a bit strange since it seems to make the whole definition self-referential. However notice that the family $\left\{L_{1}, \ldots, L_{k}\right\}$ has $k$ digraphs in it, whereas the family $\left\{L_{1}^{\prime}, \ldots, L_{k-1}^{\prime}\right\}$ only has $k-1$. Therefore the definition is consistent since first we define a linking family of size 1 , then a linking family of size 2 (using linking families of size 1), then a linking family of size 3 (using linking families of size 2), etc.

It is useful to compare a linking family of size 1 to the informal definition of linkage structures in the introduction. Given a linking family $\{L\}$ of size 1 , we see that for any pair of vertices $x, y$ outside $L$, there is a $x-y$ path using only at most 6 vertices outside of $L_{i}+x+y$. We have no control over where these extra vertices are, so they could potentially ruin the Hamiltonian cycle we are trying to build. The purpose of the paths $P_{1}, \ldots, P_{m}$ is to allow us to "protect" certain paths from being broken by these extra 6 vertices we might use when joining $x$ to $y$. We remark that the paths $P_{i}$ are allowed to consist of just one vertex in the above lemma. In this case $P_{i}^{\prime}=P_{i}$ will hold since there is only one possible path beginning and ending at the same vertex. This phenomenon can be useful since it allows us to protect a small number of vertices $\left\{v_{1}, \ldots, v_{n}\right\}$ from ever appearing in the paths $P, P_{1}^{\prime}, \ldots, P_{r}^{\prime}$ or digraphs $L_{1}^{\prime}, \ldots, L_{k-1}^{\prime}$ by letting $P_{r+1}=v_{1}, \ldots, P_{r+n}=v_{n}$.

The following is the main technical result of this paper. It shows that every highly connected tournament contains a large linking family.

Theorem 2.5. There are constants $C_{1}$ and $\Delta_{1}$ with the following property. Suppose that $T$ is a strongly $C_{1} k$-connected tournament. Then $T$ contains $k$ vertex disjoint subdigraphs $L_{1}, \ldots, L_{k}$ with maximum degree $\Delta_{1}$ and $\left|L_{1} \cup \cdots \cup L_{k}\right| \leq|T| / 100$, such that for any spanning subdigraph $D \subseteq T$ with minimum degree at least $|T|-100 \Delta_{1} k$, any subfamily $\mathcal{L} \subseteq\left\{L_{1}, \ldots, L_{k}\right\}$ is a linking family in $D \cup \mathcal{L}$. 
This Theorem is proved in Section 4. In the remainder of this section, we show how Theorem 2.5 can be used to prove Theorem 1.4.

First we'll need a simple lemma about linking families. One important feature of part (ii) of Definition 2.4 is that if $P_{1}, \ldots, P_{m}, L_{1}, \ldots, L_{k}, x$, and $y$ partition $V(D)$, then (ii) implies that $P, P_{1}^{\prime} \ldots, P_{m}^{\prime}, L_{1}^{\prime}, \ldots, L_{k-1}^{\prime}$ will partition $V(D)$ also. This allows us to obtain the following criterion for Hamiltonicity.

Lemma 2.6. Suppose that for $k \geq 1$, the vertices of a digraph $D$ can be partitioned into $k$ paths and a linking family of size $k$. Then $D$ is Hamiltonian.

Proof. The proof is by induction on $k$.

The initial case is when $k=1$. In this case we have a partition of $V(D)$ into a path $Q$ and a digraph $L$ such that $\{L\}$ is a linking family. Let $y$ and $x$ be the start and end of $Q$ respectively. Let $R=Q-x-y$. Invoking the property of linking families to the linking family $\{L\}$ with the vertices $x$ and $y$, and path $R$, we obtain two paths $P, R^{\prime}$ such that $P$ is from $x$ to $y$ and $R^{\prime}$ has the same endpoints as $R$. In addition from (ii), we have that $P$ and $R^{\prime}$ partition $V(D)$. Joining $P$ to $R^{\prime}$ produces a Hamiltonian cycle.

Now suppose that the lemma holds for $k=k_{0}$. Suppose that we have a partition of $V(D)$ into $k_{0}+1$ paths $Q_{1}, \ldots, Q_{k_{0}+1}$ and a linking family $\left\{L_{1}, \ldots, L_{k_{0}+1}\right\}$. Let $y$ and $x$ be the start and end of $Q_{k_{0}+1}$ and $Q_{k_{0}}$ respectively. Define two (possibly empty) paths $Q_{-}=Q_{k_{0}}-x$ and $Q_{+}=Q_{k_{0}+1}-y$. Invoking the property of linking families with vertices $x$ and $y$, and paths $Q_{1}, \ldots, Q_{k_{0}-1}, Q_{-}, Q_{+}$, we obtain a path $P$ from $x$ to $y$, a new linking family $\left\{L_{1}^{\prime}, \ldots, L_{k_{0}}^{\prime}\right\}$ and new paths $Q_{1}^{\prime}, \ldots, Q_{k_{0}-1}^{\prime}, Q_{-}^{\prime}, Q_{+}^{\prime}$ with the same endpoints as the previous ones. In addition $L_{1}^{\prime}, \ldots, L_{k_{0}}^{\prime}, Q_{1}^{\prime}, \ldots, Q_{k_{0}-1}^{\prime}, Q_{-}^{\prime}, Q_{+}^{\prime}$, and $P$ partition $V(D)$. Join $Q_{-}^{\prime}$ to $P$ to $Q_{+}^{\prime}$ in order to obtain a path $Q_{k_{0}}^{\prime}$. Now we have a partition of $D$ into $k$ paths $Q_{1}^{\prime}, \ldots, Q_{k_{0}}^{\prime}$ and a linking family $\left\{L_{1}^{\prime}, \ldots, L_{k_{0}}^{\prime}\right\}$. By induction, $D$ is Hamiltonian. It is worth noticing that the above proof works even when $Q^{-}$or $Q^{+}$are empty.

Combining the above lemma with Theorem 2.5 and Corollary 2.3, it is easy to prove Conjecture 1.3.

Proof of Theorem 1.4. Let $C_{1}$ and $\Delta_{1}$ be the constants in Theorem 2.5, and set $C=\left(\Delta_{1}+2\right) C_{1}$. Let $T$ be a strongly $C k^{2}$-connected tournament. Apply Theorem 2.5 in order to obtain a family of $\left(\Delta_{1}+2\right) k^{2}$ vertex disjoint subdigraphs $\left\{L_{i, j}: 1 \leq i \leq k, 1 \leq j \leq\left(\Delta_{1}+2\right) k\right\}$. Since $|T| \geq C k^{2}$, notice that from Theorem 2.5 there are at least $\left(\Delta_{1}+2\right) k$ vertices outside of $\bigcup L_{i, j}$.

Let $D_{1}$ be the digraph formed from $T$ by removing the edges of the digraphs in $\left\{L_{i, j}: 2 \leq i \leq k, 1 \leq\right.$ $\left.j \leq\left(\Delta_{1}+2\right) k\right\}$. Notice that $D_{1}$ has minimum degree $|T|-\Delta_{1}-1$ and order $\geq\left(\Delta_{1}+2\right) k$. Thus, from Theorem 2.5, the family $\left\{L_{1,1}, \ldots, L_{1,\left(\Delta_{1}+2\right) k}\right\}$ is a linking family in $D_{1}$. Apply Corollary 2.3 in order to cover $D_{1} \backslash\left(V\left(L_{1,1}\right) \cup \cdots \cup V\left(L_{1},\left(\Delta_{1}+2\right) k\right)\right)$ by $\Delta_{1}$ vertex disjoint paths. By splitting some of these paths in two we can find a partition of $D_{1} \backslash\left(V\left(L_{1,1}\right) \cup \cdots \cup V\left(L_{1,\left(\Delta_{1}+2\right) k}\right)\right)$ into exactly $\left(\Delta_{1}+2\right) k$ paths. Applying Lemma 2.6 produces a Hamiltonian cycle $C_{1}$ in $D_{1}$. Notice that since $E\left(C_{1}\right) \subseteq E\left(D_{1}\right)$, the cycle $C_{1}$ is edge disjoint from $L_{i, j}$ for $i \geq 2$.

In general, for any $\ell$ between 2 and $k$, let $D_{\ell}$ be the digraph formed from $T$ by removing the edges of all the digraphs in $\left\{L_{i, j}: \ell+1 \leq i \leq k, 1 \leq j \leq\left(\Delta_{1}+2\right) k\right\}$ and the cycles $C_{1}, \ldots, C_{\ell-1}$. Notice that $D_{\ell}$ has minimum degree $|T|-\Delta_{1}-2 \ell-1$, and so Theorem 2.5 implies that the family $\left\{L_{\ell, 1}, \ldots, L_{\ell,\left(\Delta_{1}+2\right) k}\right\}$ is a linking family in $D_{\ell}$. Apply Corollary 2.3 in order to cover $D_{\ell} \backslash\left(V\left(L_{\ell, 1}\right) \cup \cdots \cup V\left(L_{\ell,\left(\Delta_{1}+2\right) k}\right)\right)$ by $\Delta_{1}+2 \ell$ vertex disjoint paths. By splitting some of these paths in two we can find a partition of $D_{\ell} \backslash\left(V\left(L_{\ell, 1}\right) \cup \cdots \cup V\left(L_{\ell,\left(\Delta_{1}+2\right) k}\right)\right)$ into exactly $\left(\Delta_{1}+2\right) k$ paths. Applying Lemma 2.6 produces a Hamiltonian cycle $C_{\ell}$ in $D_{\ell}$. Notice that since $E\left(C_{\ell}\right) \subseteq E\left(D_{\ell}\right)$, the cycle $C_{\ell}$ is edge disjoint from $L_{i, j}$ for $i>\ell$ and $C_{t}$ for $t<\ell$.

This gives us the required edge-disjoint Hamiltonian cycles $C_{1}, \ldots, C_{k}$.

\section{Finding spanning linkages using linkage structures}

Here we prove Theorem 1.5 - that every highly connected tournament contains a spanning linkage between any two sets of $k$ vertices. The proof consists of using Theorem 2.5 to find a large linking family, and then repeatedly invoking the property of linking families in order to join $x_{i}$ to $y_{i}$ for each $i$.

Proof of Theorem 1.5. Fix $C=4 C_{1}$. Let $T$ be a $C k$-connected tournament as in the statement of Theorem 1.5. By Theorem 2.5, we have that every $C k$-connected tournament contains a family of $3 k+1$ digraphs $L_{0}, \ldots, L_{3 k}$ any subfamily of which form a linking family in $T$. Let $X=\left(x_{1}, \ldots, x_{k}\right)$ and $Y=\left(y_{1}, \ldots, y_{k}\right)$ be two disjoint sets of vertices of $T$. We will construct a spanning linkage from $X$ to $Y$.

Notice that at least $k+1$ of the graphs $L_{0}, \ldots, L_{3 k}$ must be disjoint from $\left\{x_{1}, \ldots, x_{k}, y_{1}, \ldots, y_{k}\right\}$. Without loss of generality, these are the graphs $L_{0}, \ldots, L_{k}$. Since every tournament contains a directed Hamiltonian path (this is a consequence of Redei's Theorem - see Theorem 1.4 .5 in [2]), there is a path $Q$ spanning all the 
vertices of $T$ outside $\left\{x_{1}, \ldots, x_{k}, y_{1}, \ldots, y_{k}\right\} \cup V\left(L_{0}\right) \cup \cdots \cup V\left(L_{k}\right)$. Let $y_{q}$ and $x_{q}$ be the start and end of $Q$ respectively, and let $Q^{\prime}=Q-y_{q}-x_{q}$ (the vertices $x_{q}$ and $y_{q}$ are well-defined since from Theorem 2.5 we have that $\left|L_{1} \cup \cdots \cup L_{3 k}\right| \leq|T| / 100$ and hence there are at least two vertices outside the linking family.)

Next we repeatedly apply the property of "linking families" in order to join $x_{1}$ to $x_{q}, y_{q}$ to $y_{1}, x_{2}$ to $y_{2}$, $\ldots, x_{k}$ to $y_{k}$. The following claim allows us to do this.

Claim 3.1. Suppose that for some $t \in\{1, \ldots, k+2\}$, we have a family of $t$ paths $P_{1}, \ldots, P_{t}$ and a linking family $L_{t+1}^{\prime}, \ldots, L_{k+2}^{\prime}$ such that the following hold.

(i) $P_{1}$ has the same endpoints as $Q^{\prime}$.

(ii) If $t \geq 2$, then $P_{2}$ goes from $x_{1}$ to $x_{q}$.

(iii) If $t \geq 3$, then $P_{3}$ goes from $y_{q}$ to $y_{1}$.

(iv) $P_{i+2}$ goes from $x_{i}$ to $y_{i}$ for $i=2, \ldots, t-2$.

(v) $P_{1}, \ldots, P_{t}, L_{t+1}^{\prime}, \ldots, L_{k+2}^{\prime}$, and $\left\{x_{t+1}, \ldots, x_{k}, y_{t+1}, \ldots, y_{k}\right\}$ are all vertex disjoint.

(vi) $V\left(P_{1}\right) \cup \cdots \cup V\left(P_{t}\right) \cup V\left(L_{t+1}^{\prime}\right) \cup \cdots \cup V\left(L_{k+2}^{\prime}\right) \cup\left\{x_{t+1}, \ldots, x_{k}, y_{t+1}, \ldots, y_{k}\right\}=V(T)$.

Then there is a family of $t+1$ paths $P_{1}^{\prime}, \ldots, P_{t+1}^{\prime}$ and a linking family $L_{t+2}^{\prime \prime}, \ldots, L_{k}^{\prime \prime}$ satisfying (i) - (vi) also.

Proof. For $t \geq 3$, The result is immediate from invoking the property of the linking family $\left\{L_{t+1}^{\prime}, \ldots, L_{k+2}^{\prime}\right\}$ with the vertices $x_{t+1}, y_{t+1}$, and paths $P_{1}, \ldots, P_{t}$ as well as the one-vertex paths $\left\{x_{t+2}\right\},\left\{y_{t+2}\right\}, \ldots,\left\{x_{k}\right\},\left\{y_{k}\right\}$. For $t=1$ or 2 , we do the same except with the pairs of vertices $\left(x_{1}, x_{q}\right)$ or $\left(y_{q}, y_{1}\right)$.

Starting with $t=1$ and $P_{1}=Q^{\prime}$ and the linking family $\left\{L_{0}, \ldots, L_{k}\right\}$, we apply the above claim $k+1$ times in order to obtain disjoint paths $P_{1}, \ldots, P_{k+2}$ satisfying (i) - (vi). From (vi) we know that $P_{1} \cup \cdots \cup P_{k+2}=V(T)$. We also know that $P_{1}$ has the same endpoints as $Q^{\prime}$ and so using (i) and (ii), we can join $P_{2}$ to $P_{1}$ to $P_{3}$ to get a directed path, $P$, from $x_{1}$ to $y_{1}$. Thus the family $\left\{P, P_{4}, \ldots, P_{k+2}\right\}$ is a spanning linkage from $X$ to $Y$.

\section{Linkers}

The goal of this section is to prove Theorem 2.5. We do this by constructing digraphs which we call linkers, such that any family of linkers is a linking family.

The basic idea of the construction is similar to the one introduced in [7] - the linker consists of two sets $D^{-}$ and $D^{+}$and a path $Q$ such that $D^{-}$in-dominates most vertices in the tournament, $D^{+}$out-dominates most vertices in the tournament and the path $Q$ goes from $D^{-}$to $D^{+}$. The path $P$ from $x$ to $y$ in the definition of "linking family" is then constructed by starting at $x$, then using the in-dominating property of $D^{-}$to get from $x$ to $D^{-}$, then following the path $Q$ to get to $D^{+}$, and finally using the out-dominating property of $D^{+}$ to end at $y$. The main difficulty in executing this strategy is that we won't be able to construct $D^{-}$and $D^{+}$ so that they in and out-dominate all the vertices in the tournament-instead we'll only construct them such that for every vertex $v$ in the tournament, there is a short path $P^{-}$from $x$ to $D^{-}$and a short path $P^{+}$from $D^{+}$to $x$. These paths $P^{-}$and $P^{+}$could intersect the path $Q$ in the linkage structure preventing us from using the linkage structure to join a pair of vertices together. The key idea is to begin by constructing some "spare" linkage structures which can be used to repair the path $Q$ after it is broken by $P^{-}$or $P^{+}$.

The structure of this section is as follows. In the next section we define some notation and prove some auxiliary lemmas about tournaments. In Sections 4.2 and 4.3 , we define special gadgets which we will need called dominators and connectors. Then in Section 4.4 we define linkers. In Section 4.5 we show that every highly connected tournament contains many disjoint linkers. In Section 4.6 we derive the properties of linkers which we will need. Then in Section 4.7 we put everything together and prove Theorem 2.5.

\subsection{Preliminaries}

A directed path $P$ is a sequence of vertices $v_{1}, v_{2}, \ldots, v_{k}$ in a directed graph such that $v_{i} v_{i+1}$ is an edge for all $i=1, \ldots, k-1$. All paths in this paper are directed paths. The vertex $v_{1}$ is called the start of $P$, and $v_{k}$ the end of $P$. The length of $P$ is the number of edges it has which is $|P|-1$. The vertices $v_{2}, \ldots, v_{k-1}$ are the internal vertices of $P$. Two paths are said to be internally disjoint if their internal vertices are distinct.

The out-neighbourhood of a vertex $v$ in a directed graph, denoted $N^{+}(v)$ is the set of vertices $u$ for which $v u$ is an edge. Similarly, the in-neighbourhood, denoted $N^{-}(v)$ is the set of vertices $u$ for which $u v$ is an edge. The out-degree of $v$ is $d^{+}(v)=\left|N^{+}(v)\right|$, and the in-degree of $v$ is $d^{-}(v)=\left|N^{-}(v)\right|$. A useful fact is that every 
tournament $T$ has a vertex of out-degree at least $(|T|-1) / 2$, and a vertex of in-degree at least $(|T|-1) / 2$. To see this, notice that since $T$ has $\left(\begin{array}{c}|T| \\ 2\end{array}\right)$ edges, its average in and out-degrees are both $(|T|-1) / 2$. A simple corollary of this is that every tournament has a vertex whose out-degree is neither too big nor too small.

Lemma 4.1. Every tournament $T$ on at least 10 vertices contains a vertex $v$ such that

$$
\frac{1}{5}|T| \leq d^{+}(v) \leq \frac{4}{5}|T|
$$

Proof. Let $T_{0}$ be the subtournament of $T$ consisting of vertices with out-degree $<|T| / 5$. Let $T_{1}$ be the subtournament of $T$ consisting of vertices with out-degree $>4|T| / 5$.

We know that $T_{0}$ contains a vertex $v$ such that $\left|N^{+}(v) \cap T_{0}\right| \geq\left(\left|T_{0}\right|-1\right) / 2$. Combining this with $\left|N^{+}(v)\right|<$ $|T| / 5$ gives us $\left|T_{0}\right|<2|T| / 5+1$. Similarly we obtain $\left|T_{1}\right|<2|T| / 5+1$. Therefore, using $|T| \geq 10$, there is a vertex in $T$ which is outside both $T_{0}$ and $T_{1}$, and hence satisfies the condition of the lemma.

We'll need the following definition.

Definition 4.2. A vertex $v$ in a tournament $T$ has large out-degree if there are less than $|T| / 25$ vertices $u \in T$ satisfying $d^{+}(u)>d^{+}(v)$

Vertices with large in-degree are defined similarly - a vertex has large in-degree in $T$ if there are less than $|T| / 25$ vertices $u \in T$ satisfying $d^{-}(u)>d^{-}(v)$. Notice that every tournament $T$ contains at least $|T| / 25$ vertices of large out-degree, and $|T| / 25$ vertices of large in-degree.

Recall that every tournament $T$ has a vertex of out-degree at least $(|T|-1) / 2$. By repeatedly pulling out maximum out-degree vertices, this implies that every tournament $T$ contains at least $k$ vertices of out-degree at least $(|T|-k) / 2$. Therefore, if $v$ has large out-degree in $T$, then it must satisfy $d^{+}(v) \geq 12|T| / 25$.

The important feature of vertices of large in-degrees and out-degrees is that for any pair of vertices one of which has large out-degree, and the other large in-degree, there are many short paths between them.

Lemma 4.3. Suppose that $u$ has large out-degree in $T$ and $v$ has large in-degree in $T$. Then there are at least $|T| / 25$ internally vertex disjoint paths from $u$ to $v$ in $T$, each of length at most 3 .

Proof. Let $I=N^{+}(u) \cap N^{-}(v), U=N^{+}(u) \backslash\left(N^{-}(v)+v\right)+u, \quad V=N^{-}(v) \backslash\left(N^{+}(u)+u\right)+v$, and $M$ a maximum matching of edges directed from $U$ to $V$.

Notice that there are exactly $|I|+e(M)$ paths of length $\leq 3$ from $u$ to $v$, and so if $|I|+e(M) \geq|T| / 25$ holds, then we are done. So, suppose for the sake of contradiction that we have $|I|+e(M)<|T| / 25$.

Recall that since $u$ has large out-degree we have $d^{+}(u) \geq 12|T| / 25$. This implies

$$
|U \backslash M|=\left|N^{+}(u)+u-v\right|-|I|-e(M) \geq\left|N^{+}(u)\right|-|T| / 25 \geq 11|T| / 25 \text {. }
$$

Similarly we have $|V \backslash M| \geq 11|T| / 25$. Since $N^{+}(u) \subseteq T \backslash(V+u)$ we obtain $d^{+}(u) \leq 14|T| / 25+1$.

Since $M$ is maximal, all the edges between $U \backslash M$ and $V \backslash M$ are directed from $V$ to $U$. Therefore the $|T| / 25$ vertices of largest out-degree in $V$ all have out-degree at least $|U \backslash M|+(|V \backslash M|-|T| / 25) / 2 \geq 16|T| / 25$. Since $d^{+}(u)<14|T| / 25+1$, this contradicts $u$ having large out-degree.

A tournament $T$ is transitive if for any three vertices $x, y, z \in V(T)$, if $x y$ and $y z$ are both edges, then $x z$ is also an edge. It's easy to see that a tournament is transitive exactly when it has an ordering $\left(v_{1}, v_{2}, \ldots, v_{k}\right)$ of $V(T)$ such that the edges of $T$ are $\left\{v_{i} v_{j}: i<j\right\}$. We say that $v_{1}$ is the tail of $T$, and $v_{k}$ is the head of $T$.

A simple, but very important fact is that every tournament contains a large transitive subtournament (see Exercise 1.85 in [2]).

Lemma 4.4. Every tournament $T$ contains a transitive subtournament on at $\operatorname{least} \log _{2}|T|$ vertices.

This lemma is proved by choosing the vertex sequence $\left(v_{1}, \ldots, v_{k}\right)$ of the transitive tournament recursively, by letting $v_{i}$ be a maximum out-degree vertex in the induced subtournament on $\bigcap_{j=1}^{i-1} N^{+}\left(v_{i}\right)$.

A set of vertices $S$ in-dominates another set $B$, if for every $b \in B \backslash S$, there is some $s \in S$ such that $b s$ is an edge. Notice that by this definition, a set in-dominates itself. A in-dominating set in a tournament $T$ is any set $S$ which in-dominates $V(T)$. Notice that by repeatedly pulling out vertices of largest in-degree and their in-neighbourhoods from $T$, we can find an in-dominating set of order at most $\left\lceil\log _{2}|T|\right\rceil$. 


\subsection{Dominators}

In order to construct our linking structures, we will need special sets of vertices which we call dominators. Informally dominators are small sets of vertices which dominate most of the remaining vertices in the tournament. Similar tools have been used in all previous constructions of linkage structures (see Lemma 2.3 in [6] and [10], Lemma 8.3 in [7], and Lemma 2.4 in [9]).

Dominators will come in two flavours - indominators and outdominators. Rougly speaking, an indominator consists of four transitive subtournaments $A_{1}, A_{2}, A_{3}, A_{4}$ with the crucial property that the vertices in $T$ whach are not in-dominated by $A_{2} \cup A_{3}$ all have large out-degree.

Definition 4.5. A $(m, M, p)$-indominator $D^{-}$in a tournament $T$ is a 5 -tuple $\left(A^{1}, A^{2}, A^{3}, A^{4}, E^{-}\right)$of sets of vertices in $T$ with the following properties.

(D1) $A^{1}, A^{2}, A^{3}$, and $A^{4}$ are all disjoint.

(D2) For $i=1,2,3$ the tournament on $A^{i} \cup A^{i+1}$ is transitive with tail in $A^{i}$ and head in $A^{i+1}$.

(D3) $\left|A^{2}\right|=\left|A^{3}\right|=m$.

(D4) $\left|A^{1}\right|=\left|A^{4}\right|=M$.

(D5) $A^{2} \cup A^{3}$ in-dominates $T \backslash\left(A^{1} \cup A^{2} \cup A^{3} \cup A^{4} \cup E^{-}\right)$.

(D6) $d^{+}(v) \geq p\left|E^{-}\right|$for every $v \in E^{-}$.

We call $E^{-}$the uncovered set of the indominator. The vertex set of the indominator, denoted $V\left(D^{-}\right)$is the set $A^{1} \cup A^{2} \cup A^{3} \cup A^{4}$.

We say that $D^{-}$is an $(m, M, p)$-indominator in $T$ with exceptional set $X$ if $D^{-}$is an indominator in $(T \backslash X) \cup V\left(D^{-}\right)$. This terminology will be convenient since we will sometimes have many indominators in a single tournament $T$, all of which have different exceptional sets. In the context of indominators, the set $E^{-}$ and the exceptional set $X$ play similar roles in this paper - they are both sets of vertices which may not be dominated by the indominator. The difference between these two kinds of sets is that vertices in $E^{-}$are required to have a large out-degree, whereas vertices in an exceptional set may have small out-degree.

An outdominator is defined to be an indominator in the tournament formed from $T$ by reversing all $\operatorname{arcs}$. For convenience we list its properties here.

Definition 4.6. A $(m, M, p)$-outdominator $D^{+}$in a tournament $T$ is a 5 -tuple $\left(B^{1}, B^{2}, B^{3}, B^{4}, E^{+}\right)$of sets of vertices in $T$ with the following properties.

(D1) $B^{1}, B^{2}, B^{3}$, and $B^{4}$ are all disjoint.

(D2) For $i=1,2,3$ the tournament on $B^{i} \cup B^{i+1}$ is transitive with head in $B^{i}$ and tail in $B^{i+1}$.

(D3) $\left|B^{2}\right|=\left|B^{3}\right|=m$.

(D4) $\left|B^{1}\right|=\left|B^{4}\right|=M$.

(D5) $B^{2} \cup B^{3}$ out-dominates $T \backslash\left(B^{1} \cup B^{2} \cup B^{3} \cup B^{4} \cup E^{+}\right)$.

(D6) $d^{-}(v) \geq p\left|E^{+}\right|$for every $v \in E^{+}$.

When dealing with indominators, they will always be labelled by " $D^{-}$" (possibly with some subscript), their four sets of vertices will always be labelled by " $A^{1}, \ldots, A^{4}$ ", and the set of uncovered vertices will be labelled " $E^{-}$". Similarly outdominators will always be labelled as in their definition. Exceptional sets of vertices will always be labelled by the letter " $X$ ". The tail of the transitive tournament on $A^{1}$ in an indominator $D^{-}$is called the tail of $D^{-}$, and the head of $A^{4}$ is the head of $D^{-}$. Similarly in an outdominator $D^{+}$, the head of $B^{1}$ and the tail of $B^{4}$ are called the head and tail of $D^{+}$respectively.

The following lemma is an intermediate step we need in order to construct dominators.

Lemma 4.7. For any numbers $m, M, L$, and $p$ with $L \geq 4 \cdot 5^{m+M}$ and $p \leq(5 / 4)^{m-1}$, the following holds. If $T$ is a tournament with $|T|>L$, then there are sets of vertices $A, B, E^{-}, X \subseteq V(T)$ with the following properties.

(i) $A \cup B$ is a transitive tournament with its tail in $A$ and its head in $B$ 
(ii) $|A|=m,|B|=M$.

(iii) $A$ in-dominates $T \backslash\left(E^{-} \cup X\right)$.

(iv) $|X| \leq L$.

(v) $d^{+}(u) \geq p\left|E^{-}\right|$for every vertex $u \in E^{-}$.

Proof. Let $X_{0}$ be the set of vertices in $T$ of out-degree less than $2 \cdot 5^{m+M}$. Notice that since $X_{0}$ contains a vertex with out-degree at least $\left(\left|X_{0}\right|-1\right) / 2$, we must have that $\left|X_{0}\right| \leq 4 \cdot 5^{m+M}$.

Let $v_{0}$ be a vertex contained in $T \backslash X_{0}$ of minimal out-degree. Since $v_{0}$ is not in $X_{0}$, we have $d^{+}\left(v_{0}\right) \geq$ $2 \cdot 5^{m+M}$. Notice also that by our choice of $v_{0}$, every $y \in T \backslash X_{0}$ must satisfy $\left|N^{+}(y)\right| \geq\left|N^{+}\left(v_{0}\right)\right|$.

For $i=1, \ldots, m+M$, let $v_{i}$ be any vertex in $\bigcap_{j=0}^{i-1} N^{+}\left(v_{j}\right)$ satisfying

$$
\frac{1}{5}\left|\bigcap_{j=0}^{i-1} N^{+}\left(v_{j}\right)\right| \leq\left|N^{+}\left(v_{i}\right) \cap\left(\bigcap_{j=0}^{i-1} N^{+}\left(v_{j}\right)\right)\right| \leq \frac{4}{5}\left|\bigcap_{j=0}^{i-1} N^{+}\left(v_{j}\right)\right| .
$$

We can always choose such a vertex by Lemma 4.1, as long as $\bigcap_{j=0}^{i-1} N^{+}\left(v_{j}\right)$ has size at least 10 . This holds since by the construction we always have $\left|\bigcap_{j=0}^{i-1} N^{+}\left(v_{j}\right)\right| \geq\left(\frac{1}{5}\right)^{i-1}\left|N^{+}\left(v_{0}\right)\right|$ which is greater than 10 for $i=1, \ldots, m+M$ (using $\left.\left|N^{+}\left(v_{0}\right)\right| \geq 2 \cdot 5^{m+M}\right)$. Notice that we have $\left|\bigcap_{i=0}^{m-1} N^{+}\left(v_{i}\right)\right| \leq\left(\frac{4}{5}\right)^{m}\left|N^{+}\left(v_{0}\right)\right|$.

Now, we set $A=\left\{v_{0}, \ldots, v_{m-1}\right\}, \quad B=\left\{v_{m}, \ldots, v_{m+M-1}\right\}, \quad E^{-}=\left(\bigcap_{i=0}^{m-1} N^{+}\left(v_{i}\right)\right) \backslash X_{0}, \quad$ and $\quad X=$ $\left(\bigcap_{i=0}^{m-1} N^{+}\left(v_{i}\right)\right) \cap X_{0}$. Conditions (i) - (iv) are immediate with this choice. For condition (v), notice that for $y \in E^{-}$we have

$$
d^{+}(y) \geq d^{+}\left(v_{0}\right) \geq\left(\frac{5}{4}\right)^{m}\left|\bigcap_{i=0}^{m-1} N^{+}\left(v_{i}\right)\right| \geq\left(\frac{5}{4}\right)^{m}\left|E^{-}\right| .
$$

The following lemma guarantees the existence of dominators in tournaments.

Lemma 4.8. For any numbers $m, M, L$, and $p$ with $L \geq 4 \cdot 5^{m+M}+m+M$ and $p \leq \frac{1}{2}(5 / 4)^{m-1}$, the following holds. Let $T$ be a tournament on at least $25 \cdot 2^{2 m+2 M}$ vertices and $Y \subseteq V(T)$ with $|Y| \leq|T| / 25-2^{2 m+2 M}$. Then, $T$ contains a $(m, M, p)$-indominator $D^{-}=\left(A^{1}, A^{2}, A^{3}, A^{4}, E^{-}\right)$with an exceptional set $X$ such that $Y \cap V\left(D^{-}\right)=\emptyset, Y \subseteq X,|X| \leq L+|Y|$, and all vertices of $A^{1}$ have large in-degree in $T$.

Proof. Notice that since $|T| \geq 25 \cdot 2^{2 m+2 M}$ there are at least $2^{2 m+2 M}$ vertices in $T \backslash Y$ which have large indegree in $T$. Therefore, by Lemma 4.4, we can choose a transitive subtournament $S \subseteq T \backslash Y$ of $2 m+2 M$ vertices with large in-degree. Let $S_{1}$ be the first $M$ vertices of $S, S_{2}$ the next $m$ vertices, $S_{3}$ the next $m$ vertices, and $S_{4}$ the last $M$ vertices. Let $T^{\prime}=T \backslash\left(Y \cup S_{1} \cup S_{2} \cup S_{3} \cup S_{4} \cup N^{-}\left(S_{2}\right)\right)$.

If $\left|T^{\prime}\right| \leq L$, then the lemma follows by choosing $A^{i}=S_{i}$ for $i=1,2,3,4, E^{-}=\emptyset$, and $X=V\left(T^{\prime}\right) \cup Y$.

If $\left|T^{\prime}\right| \geq L$, then we apply Lemma 4.7 to $T^{\prime}$ with the parameters $m, M, p$, and $L^{\prime}=L-M-m$. This gives us sets $A, B, E^{-}$, and $X$ as in Lemma 4.7 satisfying $|A|=m,|B|=M$, and $|X| \leq L-M-m$. Then we let $A^{1}=S_{1}, A^{2}=S_{2}, A^{3}=A, A^{4}=B$, and $X^{\prime}=X \cup Y \cup S_{3} \cup S_{4}$. With this definition $\left(A^{1}, A^{2}, A^{3}, A^{4}, E^{-}\right)$, is an indominator in $T$ with exceptional set $X^{\prime}$. Indeed, conditions (D1) - (D5) are immediate, and part (v) of Lemma 4.7 implies that (D6) holds.

By reversing arcs, we obtain the following version of Lemma 4.8 for outdominators.

Lemma 4.9. For any numbers $m, M, L$, and $p$ with $L \geq 4 \cdot 5^{m+M}+m+M$ and $p \leq \frac{1}{2}(5 / 4)^{m-1}$, the following holds. Let $T$ be a tournament on at least $25 \cdot 2^{2 m+2 M}$ vertices and $Y \subseteq V(T)$ with $|Y| \leq|T| / 25-2^{2 m+2 M}$. Then, $T$ contains a $(m, M, p)$-outdominator $D^{+}=\left(B^{1}, B^{2}, B^{3}, B^{4}, E^{+}\right)$with an exceptional set $X$ such that $Y \cap V\left(D^{+}\right)=\emptyset, Y \subseteq X,|X| \leq L+|Y|$, and all vertices of $B^{1}$ have large out-degree in $T$. 
Given an indominator $D^{-}$in a tournament $T$, we will sometimes want to modify $T$, and still know that $D^{-}$is an indominator in the modified tournament. If $D^{-}$has exceptional set $X$, then from the definition of "exceptional set," we see that removing any vertices of $X \backslash V\left(D^{-}\right)$from $T$ will preserve $D^{-}$being an indominator. Similarly, we can remove two sets of vertices of the same size from $X \cap A_{1}$ and $X \cap A_{4}$ to obtain a new indominator. Corresponding results hold for outdominators as well.

Given a dominator $D$ with exceptional set $X$, we will sometimes want to increase the size of $X$ and still know that $D$ is a dominator with the larger exceptional set. The following lemma allows us to do this under the assumption that $T$ has large degree.

Lemma 4.10. Let $T$ be a tournament of minimum out-degree $\delta^{+}(T)$, and $D^{-}$an $(m, M, p)$-indominator in $T$ with exceptional set $X$. For any $Y$ satisfying $X \subseteq Y$ and $2|Y| \leq \delta^{+}(T), D^{-}$is an $(m, M, p / 2)$-indominator in $T$ with exceptional set $Y$.

Proof. The only part of the definition of an indominator which needs checking is (D6). Let $E^{-}$be the set of uncovered vertices of $D^{-}$in $T \backslash X$, and $v$ be a vertex in $E^{-}$. We need to show that $\left|N^{+}(v) \backslash Y\right| \geq p\left|E^{-} \backslash Y\right| / 2$.

Since $D^{-}$is an $(m, M, p)$-indominator in $T$ with exceptional set $X$, we have $d^{+}(v) \geq p\left|E^{-}\right|$. We also have $d^{+}(v) \geq 2|Y|$. Averaging these gives $d^{+}(v) \geq p\left|E^{-}\right| / 2+|Y|$. This implies the result

$$
\left|N^{+}(v) \backslash Y\right| \geq d^{+}(v)-|Y| \geq p\left|E^{-}\right| / 2 \geq p\left|E^{-} \backslash Y\right| / 2
$$

By reversing arcs in the above lemma, we obtain the following version of it for outdominators

Lemma 4.11. Let $T$ be a tournament of minimum in-degree $\delta^{-}(T)$, and $D^{+}$an $(m, M, p)$-outdominator in $T$ with exceptional set $X$. For any $Y$ satisfying $X \subseteq Y$, and $2|Y| \leq \delta^{-}(T), D^{+}$is an $(m, M, p / 2)$-outdominator in $T$ with exceptional set $Y$.

\subsection{Connectors}

In order to construct our linking structures, we will need special gadgets which we call "connectors". Informally, a connector is a small set of vertices together with two coverings of it - one by four paths, and one by five.

Definition 4.12. A connector is any digraph $C$ on at most 40 vertices and containing distinct vertices $x_{1}, \ldots, x_{5}, y_{1}, \ldots, y_{5}$ with the following property. For $n \in\{4,5\}$, there are vertex disjoint paths $P_{1}, \ldots, P_{n}$ such $P_{i}$ is from $x_{i}$ to $y_{i}$, and $V(C)=V\left(P_{1}\right) \cup \cdots \cup V\left(P_{n}\right)$.

The vertices $x_{1}, \ldots, x_{5}$ are the sources of the connector and the vertices $y_{1}, \ldots, y_{5}$ are the sinks of the connector.

One example of a connector is a transitive tournament $T$ on 10 vertices, with vertex sequence $x_{1}, x_{2}, x_{3}, x_{5}, x_{4}, y_{1}, y_{2}, y_{3}, y_{5}, y_{4}$. Its easy to see that for $n=4$ or 5 , we can find $n$ disjoint $x_{i}-y_{i}$ paths covering $T$. For our purposes, we'll need slightly more complicated connectors. The following lemma allows us to find a connector with prescribed sources and sinks under certain conditions.

Lemma 4.13. There is a constant $N=99 \cdot 2^{2^{2^{2^{10}}}}$ such that the following holds. Let $T$ be a tournament on at least $200 N$ vertices, $Y$ a set of vertices in $T$ with $|Y| \leq|T| / 50$, and $\left\{x_{1}, \ldots, x_{N}, y_{1}, \ldots, y_{N}\right\}$ a set of $2 N$ vertices in $T \backslash Y$ such that $x_{1}, \ldots, x_{N}$ have large out-degree in $T$, and $y_{1}, \ldots, y_{N}$ have large in-degree in $T$. Then there is a connector $C$ contained in $T \backslash Y$, such that the sources of $C$ are in $\left\{x_{1}, \ldots, x_{N}\right\}$, and the sinks in $\left\{y_{1}, \ldots, y_{N}\right\}$.

Proof. Lemma 4.3 implies that for any $i=1, \ldots, N$ there are at least $|T| / 25$ internally vertex disjoint paths of length at most 3 from $x_{i}$ to $y_{i}$. Since $|Y| \leq|T| / 50$, there are at least $|T| / 50$ such paths avoiding $Y$. Therefore, using $|T| \geq 200 N$, we can choose vertex disjoint paths $P_{1}, \ldots, P_{N}$ of length at most 3 in $T \backslash Y$, such that $P_{i}$ is from $x_{i}$ to $y_{i}$.

Notice that at least a third of these paths must have the same length. Without loss of generality we can assume that the paths $P_{1}, \ldots, P_{N / 3}$ all have $\ell$ vertices with $\ell \leq 4$.

For $i=1, \ldots, N / 3$, let $p_{i}^{1}, \ldots, p_{i}^{\ell}$ be the vertex sequence of $P_{i}$ (so we have $p_{i}^{1}=x_{i}$ and $p_{i}^{\ell}=y_{i}$ ). By Lemma 4.4, there is some subset $I_{1} \subseteq[N / 3]$ with $\left|I_{1}\right| \geq \log _{2} N / 3$ such that the subtournament on $\left\{p_{i}^{1}: i \in I_{1}\right\}$ is transitive. Applying Lemma 4.4 again, we find some subset $I_{2} \subseteq I_{1}$ with $\left|I_{2}\right| \geq \log _{2} I_{1}$ such that the subtournaments on $\left\{p_{i}^{2}: i \in I_{2}\right\}$ and $\left\{p_{i}^{1}: i \in I_{2}\right\}$ are both transitive. Applying Lemma $4.4 \ell-2$ more times 


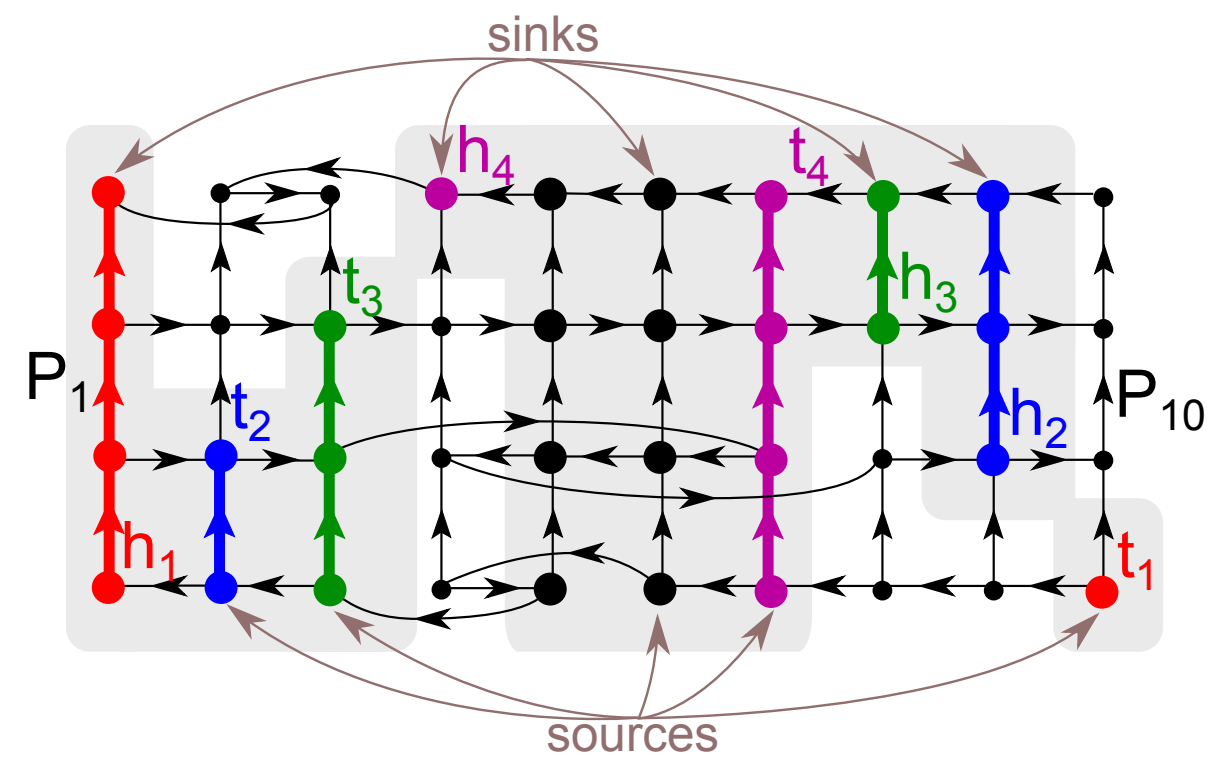

Fig. 1. The subtournament $T_{1}$. In this diagram $\ell=4$. The vertical lines are the paths $P_{1}, \ldots, P_{10}$. The horizontal lines show the order of the vertices of the transitive tournaments $\left\{p_{i}^{k}: i \in I_{k}\right\}$ for $k=1, \ldots, 4$. The coloured lines are the paths $P_{h}^{\prime 1}, \ldots, P_{h}^{\prime 4}, P_{t}^{\prime 1}, \ldots, P_{t}^{\prime 4}$. The grey area represents the vertices which are in the connector $C$.

we obtain a subset $I_{\ell} \subseteq I_{2}$ with $\left|I_{\ell}\right| \geq \log _{2} \log _{2} \log _{2} \log _{2} N / 3 \geq 10$ such that the subtournaments on $\left\{p_{i}^{j}: i \in I_{\ell}\right\}$ are transitive for $j=1, \ldots, \ell$. Without loss of generality, we can suppose that $I_{\ell}$ contains the set $\{1, \ldots, 10\}$.

For each $j=1, \ldots, \ell$, we define a subtournament $T_{j}$, vertices $h_{j}$ and $t_{j}$, and two paths $P_{h}^{j}$ and $P_{t}^{j}$ as follows: Let $T_{1}$ be the subtournament of $T$ on vertices $V\left(P_{1}\right) \cup \cdots \cup V\left(P_{10}\right)$. Let $h_{1}$ and $t_{1}$ be the head and tail respectively of the transitive tournament on $\left\{p_{i}^{1}: 1 \leq i \leq 10\right\}$. Let $P_{h}^{1}$ and $P_{t}^{1}$ be the $P_{i}$-paths containing $h_{1}$ and $t_{1}$ respectively. Then, for $j=2, \ldots, \ell$, let $T_{j}=T_{j-1} \backslash\left(P_{h}^{j-1} \cup P_{t}^{j-1}\right)$. Let $h_{j}$ and $t_{j}$ be the head and tails respectively of the transitive tournament on $T_{j} \cap\left\{p_{i}^{j}: 1 \leq i \leq 10\right\}$. Let $P_{h}^{j}$ and $P_{t}^{j}$ be the $P_{i}$-paths containing $h_{j}$ and $t_{j}$ respectively.

For $j=1, \ldots, \ell$, let $P_{h}^{\prime j}$ be the final segment of the path $P_{h}^{j}$ starting from $h_{j}$, and let $P_{t}^{\prime j}$ be the initial segment of the path $P_{t}^{j}$ ending at $t_{j}$. See Figure 1 for a diagram of the paths $P_{h}^{\prime 1}, \ldots, P_{h}^{\prime 4}, P_{t}^{\prime 1}, \ldots, P_{t}^{\prime 4}$ being constructed.

We can now define the connector $C$. Let $C$ the subtournament of $T$ on the vertices $\left(T_{1} \backslash \bigcup_{j=1}^{\ell} P_{h}^{j} \cup P_{t}^{j}\right) \cup$ $\left(\bigcup_{j=1}^{\ell} P_{h}^{\prime j} \cup P_{t}^{\prime j}\right)$. In other words $C$ is the tournament on the vertices of $T_{1}$ with all the paths $P_{h}^{j}$ and $P_{t}^{j}$ removed, but then with the initial and final segments $P_{h}^{\prime j}$ and $P_{t}^{\prime j}$ added back in. Notice that we have $|C| \leq\left|T_{1}\right| \leq 40$. Let $y_{1}^{\prime}, \ldots, y_{\ell}^{\prime}$ be the ends of the paths $P_{h}^{\prime 1}, \ldots, P_{h}^{\prime \ell}$. Let $x_{1}^{\prime}, \ldots, x_{\ell}^{\prime}$ be the starts of the paths $P_{t}^{\prime 1}, \ldots, P_{t}^{\prime \ell}$. Notice that since $\ell \leq 4$, there must be at least $5-\ell$ paths in $\left\{P_{1}, \ldots, P_{10}\right\}$ which are vertex disjoint from $P_{h}^{\prime 1} \ldots, P_{h}^{\prime \ell}, P_{t}^{\prime 1} \ldots, P_{t}^{\prime \ell}$. Let $x_{\ell+1}^{\prime}, \ldots, x_{5}^{\prime}$ be the starts of any choice of such paths. Let $y_{\ell+1}^{\prime}, \ldots, y_{5}^{\prime}$ be the ends of the paths containing $x_{\ell+1}^{\prime}, \ldots, x_{5}^{\prime}$.

We claim that $C$ is a connector with sources $x_{1}^{\prime}, \ldots, x_{5}^{\prime}$ and $\operatorname{sinks} y_{1}^{\prime}, \ldots, y_{5}^{\prime}$. To see this, let $n=4$ or 5 . For $i=\ell+1, \ell+2, \ldots, n$, let $P_{i}^{\prime}$ be the path between $x_{i}^{\prime}$ and $y_{i}^{\prime}$ (which is one of the paths in $\left\{P_{1}, \ldots, P_{10}\right\}$ ). For $i=1, \ldots, \ell$, let $R_{i}$ be a path from $t_{i}$ to $h_{i}$ consisting of all the vertices in $V(C) \cap\left\{p_{t}^{i}: 1 \leq t \leq 10\right\} \backslash\left(V\left(P_{\ell+1}^{\prime}\right) \cup\right.$ $\cdots \cup V\left(P_{n}^{\prime}\right)$ ) (such a path exists because $V(C) \cap\left\{p_{t}^{i}: 1 \leq t \leq 10\right\}$ is a transitive tournament with head $h_{i}$ and tail $t_{i}$ ). For $i=1, \ldots, \ell$, let $P_{i}^{\prime}$ be the path formed by joining $P_{h}^{\prime i}$ to $R_{i}$ to $P_{t}^{\prime i}$. Now, we have that for each $i=1, \ldots, n, P_{i}^{\prime}$ goes from $x_{i}^{\prime}$ to $y_{i}^{\prime}$, and $V(C)=V\left(P_{1}^{\prime}\right) \cup \cdots \cup V\left(P_{n}^{\prime}\right)$ as required.

\subsection{Definition of linkers}

Here we define our linkage structures. Informally a linker consists of several indominators, outdominators, and connectors together with a collection of paths connecting them all together. See Figure 2 for an illustration of a linker.

Definition 4.14. A $t$-linker $L$ in $T$ consists of $t(32,32,32)$-indominators $D_{1}^{-}, \ldots, D_{t}^{-}$with $D_{i}^{-}=\left(A_{i}^{1}, A_{i}^{2}, A_{i}^{3}\right.$, $\left.A_{i}^{4}, E_{i}^{-}\right), t(32,32,32)$-outdominators $D_{1}^{+}, \ldots, D_{t}^{+}$with $D_{i}^{+}=\left(B_{i}^{1}, B_{i}^{2}, B_{i}^{3}, B_{i}^{4}, E_{i}^{+}\right), t$ connectors $C_{1}, \ldots C_{t}$, $5 t$ directed paths $Q_{1}, \ldots, Q_{5 t}$, and a set $X$ which have the following properties. 


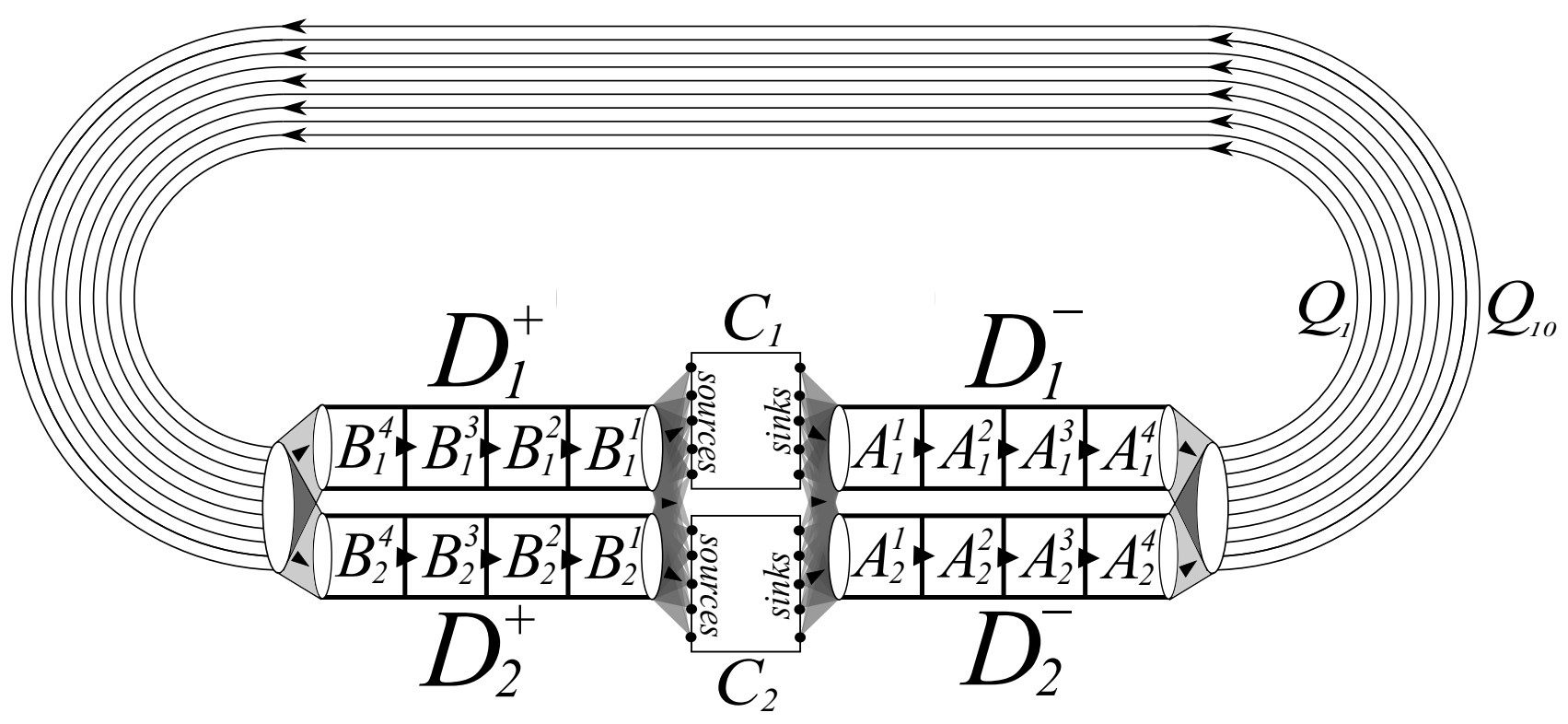

Fig. 2. A 2-linker

(L1) The indominators $D_{1}^{-}, \ldots, D_{t}^{-}$, outdominators $D_{1}^{+}, \ldots, D_{t}^{+}$, paths $Q_{1}, \ldots, Q_{5 t}$, and connectors, $C_{1}, \cdots C_{t}$ are all vertex disjoint.

(L2) The indominators $D_{1}^{-}, \ldots, D_{t}^{-}$and outdominators $D_{1}^{+}, \ldots, D_{t}^{+}$all have the common exceptional set $X$. We have $V\left(D_{i}^{-}\right), V\left(D_{i}^{+}\right), V\left(C_{i}\right) \subseteq X$ for all $i=1, \ldots, t$.

(L3) We have $\left|E_{1}^{-}\right| \geq\left|E_{2}^{-}\right| \geq \cdots \geq\left|E_{t}^{-}\right|$and $\left|E_{1}^{+}\right| \geq\left|E_{2}^{+}\right| \geq \cdots \geq\left|E_{t}^{+}\right|$.

(L4) Either $\left|E_{t}^{-}\right| \geq\left|E_{1}^{+}\right|$or $\left|E_{t}^{+}\right| \geq\left|E_{1}^{-}\right|$holds.

(L5) For all $i$ and $j$, the following directed edges are present:

- Every edge from the sinks of $C_{j}$ to any vertex in $A_{i}^{1}$.

- Every edge from any vertex in $B_{i}^{1}$ to the sources of $C_{j}$.

- Every edge from any vertex in $A_{i}^{4}$ to the start of $Q_{j}$.

- Every edge from the end of $Q_{j}$ to any vertex in $B_{i}^{4}$.

The vertices of $L$ are $V(L)=\left(\bigcup_{i=1}^{5 t} V\left(Q_{i}\right)\right) \cup\left(\bigcup_{i}^{t} V\left(D_{i}^{-}\right) \cup V\left(D_{i}^{+}\right) \cup V\left(C_{i}\right)\right)$. The vertices inside $\bigcup_{i}^{t} V\left(D_{i}^{-}\right) \cup V\left(D_{i}^{+}\right) \cup V\left(C_{i}\right)$ are called the essential vertices of the $t$-linker. The vertices in $Q_{1}, \ldots, Q_{5 t}$ are called the path vertices of the $t$-linker. The edges of $L$ are all the edges contained in the dominators $D_{i}^{-}, D_{i}^{+}$, connectors $C_{i}$, paths $Q_{i}$, as well as all the edges mentioned in (L5). One important observation is that the union of all the edges of a $t$-linker has maximum degree $\leq 32 t+40$ (which holds since the maximum degree vertices in the linker are the sources and sinks of the connectors).

The set $X$ is called the exceptional set of the $t$-linker. Notice that if $L$ is a linker in $T$ with exceptional set $X$, then removing any vertices of $X \backslash V(L)$ from $T$ produces a new tournament $T^{\prime}$ where $L$ is still a linker with exceptional set $X \cap T^{\prime}$.

It is worth noticing that if $L$ is a $t$-linker in a tournament $T$, then it will also be a $t$-linker in the tournament $T^{o p}$ produced from $T$ by reversing all arcs (where we also exchange the roles of the indominators and outdominators in $L$ ). This will be useful since in some circumstances it allows us to assume that $E_{t}^{-} \geq E_{1}^{+}$ occurs in (L4) without losing generality.

One useful property that $t$-linkers have is that it is possible to partition them into smaller linkers -indeed for any integers satisfying $t=t_{1}+\ldots, t_{k}$ the vertices of any $t$-linker $L$ with exceptional set $X$ can be partitioned into $L_{1}, \ldots, L_{k}$ where for each $i, L_{i}$ is a $t_{i}$-linker with exceptional set $X$. To see this note that choosing $L_{i}$ to consist of any choice of $5 t_{i}$ paths in $L$ and $t_{i}$ indominators, outdominators, and connectors in $L$ gives a $t_{i}$-linker. 


\subsection{Construction of linkers}

The aim of this section is to show that for every $t$, there is a constant $C_{0}=C_{0}(t)$ such that every $C_{0} k$-connected tournament contains $k$ vertex disjoint $t$-linkers. The overall strategy is to use Lemmas $4.8,4.9$, and 4.13 to find many indominators, outdominators, and connectors in $T$ and then use Menger's Theorem to find the paths $Q_{i}$.

The following lemma will be used in our construction of linkers in order to ensure that (L5) holds.

Lemma 4.15. For all $m, t, \ell \in \mathbb{N}$ there exists $R(m, t, \ell) \in \mathbb{N}$ such that the following holds. Suppose that $A_{1}, \ldots, A_{R}$ are disjoint sets of vertices of order $2 m$ in a tournament $T$. Then we can choose disjoint sets $I, J \subseteq[R]$, subsets $A_{i}^{\prime} \subseteq A_{i}$ for all $i \in I$, and vertices $v_{j} \in A_{j}$ for all $j \in J$ with the following properties.

- We have $|I|=t$ and $|J|=\ell$, and $\left|A_{i}^{\prime}\right|=m$ for all $i$.

- For all $i, j$, all the edges between $A_{i}^{\prime}$ and $v_{j}$ are directed from $A_{i}^{\prime}$ to $v_{j}$.

Proof. If $R_{k}(n)$ denotes the $k$-colour Ramsey number of the complete graph on $n$ vertices, let $R=R(m, t, \ell)=$ $R_{2 m\left(\begin{array}{c}2 m \\ m\end{array}\right)}\left(2^{t+\ell}\right)$.

For each $i$, let the vertices of $A_{i}$ be called $a_{i}^{1}, \ldots, a_{i}^{2 m}$. Notice that for any $i \neq j$ there must be a vertex in either $A_{i}$ or $A_{j}$ which has in-degree at least $m$ in the bipartite digraph between $A_{i}$ and $A_{j}$. For every $i, j$ choose one such vertex, which we call $v_{i, j}$ and let $N_{i, j}$ be some particular subset of order $m$ of the in-neighbourhood of $v_{i, j}$ in the bipartite digraph between $A_{i}$ and $A_{j}$.

We define a coloured tournament $S$ whose vertex set is $\{1, \ldots, R\}$. The edge between $i$ and $j$ in $S$ is directed $i j$ if $v_{i, j} \in A_{j}$ holds and $j i$ if $v_{i, j} \in A_{i}$ holds. In addition we give each edge in $S$ one of $2 m\left(\begin{array}{c}2 m \\ m\end{array}\right)$ colours which are indexed by the set $[2 m] \times\left(\begin{array}{c}{[2 m]} \\ m\end{array}\right)$. We let the edge $i j$ have colour $(t, X)$ if $v_{i, j}=a_{j}^{t}$ and $N_{i, j}=\left\{a_{i}^{x}: x \in X\right\}$.

By Ramsey's Theorem, combined with Lemma 4.4, there is a monochromatic transitive subtournament $S^{\prime}$ of $S$ on $t+\ell$ vertices. Let $x_{1}, x_{2}, \ldots, x_{t+\ell}$ be the vertex sequence of $S^{\prime}$ in the order from head to tail. Let $I=\left\{x_{1}, \ldots, x_{t}\right\}$ and $J=\left\{x_{t+1}, \ldots, x_{t+\ell}\right\}$. The edges in $S^{\prime}$ all have the same colour $(t, X) \in[2 m] \times\left(\begin{array}{c}{[2 m]} \\ m\end{array}\right)$. For each $i \in I$ we let $A_{i}^{\prime}=\left\{a_{i}^{x}: x \in X\right\}$, and for each $j \in J$ we let $v_{j}=v_{j}^{t}$. By the definition of the coloured tournament $S$, this choice of $I, J, A_{i}^{\prime} \mathrm{s}$, and $v_{j}$ s satisfy all the conditions of the lemma.

By reversing arcs in the above lemma, we get the following.

Lemma 4.16. For all $m, t, \ell \in \mathbb{N}$ there exist $R(m, t, l) \in \mathbb{N}$ such that the following holds. Suppose that $A_{1}, \ldots, A_{R}$ are disjoint sets of vertices of order $2 m$ in a tournament $T$. Then we can choose disjoint sets $I, J \subseteq[R]$, subsets $A_{i}^{\prime} \subseteq A_{i}$ for all $i \in I$, and vertices $v_{j} \in A_{j}$ for all $j \in J$ with the following properties.

- We have $|I|=t$ and $|J|=\ell$, and $\left|A_{i}^{\prime}\right|=m$ for all $i$.

- For all $i, j$, all the edges between $A_{i}^{\prime}$ and $v_{j}$ are directed from $v_{j}$ to $A_{i}^{\prime}$.

The following technical lemma allows us to find a single $t$-linker in a tournament assuming that we have many disjoint in and out-dominators with paths between them.

Lemma 4.17. For every $t \in \mathbb{N}$, there is a constant $R_{0}=R_{0}(t)$ such that the following holds. Suppose that $T$ is a tournament, $X$ and $Z$ are subsets of $V(T)$, and $H_{1}, \ldots, H_{R_{0}}$ are vertex disjoint subdigraphs of $T$ with the following properties.

(i) $H_{i}$ consists of:

- An $(32,64,64)$-indominator $D_{i}^{-}=\left(A_{i}^{1}, A_{i}^{2}, A_{i}^{3}, A_{i}^{4}, E_{i}^{-}\right)$of $T$ with exceptional set $X$.

- An $(32,64,64)$-outdominator $D_{i}^{+}=\left(B_{i}^{1}, B_{i}^{2}, B_{i}^{3}, B_{i}^{4}, E_{i}^{+}\right)$of $T$ with exceptional set $X$.

- A path $Q_{i}$ from the head of $D_{i}^{-}$to the tail of $D_{i}^{+}$.

In addition, $D_{i}^{-}, D_{i}^{+}$, and the internal vertices of $Q_{i}$ are all vertex disjoint for each $i$.

(ii) All vertices in $A_{i}^{1}$ have large in-degree in $T$. All vertices in $B_{i}^{1}$ have large out-degree in $T$.

(iii) $V\left(D_{i}^{-}\right), V\left(D_{i}^{+}\right) \subseteq X$ and $V\left(H_{i}\right) \subseteq Z$ hold for all $i$.

(iv) $|Z| \leq|T| / 50-40 t$.

(v) $2(|X|+40 t) \leq \min \left(\delta^{+}(T), \delta^{-}(T)\right)$. 
Then there is a set of vertices $S$ with $|S| \leq 40 t$ and $S \cap Z=\emptyset$, and a $t$-linker $L$ with exceptional set $X \cup S$ whose vertices are contained in $S \cup V\left(H_{1}\right) \cup \cdots \cup V\left(H_{R_{0}}\right)$. In addition, for any $Y$ containing $X \cup S$ with $2|Y| \leq \min \left(\delta^{+}(T), \delta^{-}(T)\right), L$ is also a $t$-linker in $T$ with exceptional set $Y$.

Proof. Let $N$ be the constant from Lemma 4.13. Let $R(m, t, \ell)$ be the function given by Lemma 4.15 . We fix the following constants for the proof.

$$
\begin{aligned}
& R_{2}=R(32,2 t, N+40 t) \\
& R_{1}=R\left(32, R_{2}, 5 t\right) \\
& R_{0}=R\left(32, R_{2}, R_{1}\right)
\end{aligned}
$$

Apply Lemma 4.15 to the family $\left\{A_{1}^{4}, \ldots, A_{R_{0}}^{4}\right\}$ in order to find disjoint sets $I_{D^{-}, C_{\text {sink }}}$ and $J_{D^{-}, Q, C_{\text {source }}}$ such that $\left|I_{D^{-}, C_{s i n k}}\right|=R_{2},\left|J_{D^{-}, Q, C_{\text {source }}}\right|=R_{1}$, and also for each $i \in I_{D^{-}, C_{s i n k}}$ there is an $A_{i}^{\prime 4} \subseteq A_{i}^{4}$ of order 32 , and for each $j \in J_{D^{-}, Q, C_{\text {source }}}$ there is a vertex $v_{j}^{-} \in A_{j}^{4}$. In addition we have all the edges going from $A_{i}^{\prime 4}$ to $v_{j}^{-}$for any $i \in I_{D^{-}, C_{s i n k}}$ and $j \in J_{D^{-}, Q, C_{\text {source }}}$.

Apply Lemma 4.16 to the family $\left\{B_{j}^{4}: j \in J_{D^{-}, Q, C_{\text {source }}}\right\}$ in order to find disjoint sets $I_{D^{+}, C_{\text {source }}}$ and $J_{Q}$ contained in $J_{D^{-}, Q, C_{\text {source }}}$ such that $\left|I_{D^{+}, C_{\text {source }}}\right|=R_{2},\left|J_{Q}\right|=5 t$, and also for each $i \in I_{D^{+}, C_{\text {source }} \text { there is an }}$ $B_{i}^{\prime 4} \subseteq B_{i}^{4}$ of order 32 , and for each $j \in J_{Q}$ there is a vertex $v_{j}^{+} \in B_{j}^{4}$. In addition we have all the edges going from $v_{j}^{+}$to $B_{i}^{\prime 4}$ for any $i \in I_{D^{+}, C_{\text {source }}}$ and $j \in J_{Q}$.

Notice that for each $j \in J_{Q}$ the fact that $A_{j}^{4}$ is a transitive tournament implies that there is an edge from $v_{j}^{-}$to the start of $Q_{j}$. Similarly, there is an edge in $B_{j}^{4}$ from the end of $Q_{j}$ to $v_{j}^{+}$. Joining these two edges to $Q_{j}$, we obtain a path $Q_{j}^{\prime}=v_{j}^{-}+Q_{j}+v_{j}^{+}$. For all $i \in I_{D^{-}, C_{s i n k}}, i^{\prime} \in I_{D^{+}, C_{\text {source }}}$, and $j \in J_{Q}$ all the edges between $A_{i}^{4}$ and the start of $Q_{j}^{\prime}$ are oriented towards $Q_{j}^{\prime}$ and all edges between the end of $Q_{j}^{\prime}$ and $B_{i^{\prime}}^{4}$ are oriented towards $B_{i^{\prime}}^{4}$.

Apply Lemma 4.15 to the family $\left\{B_{i}^{1}: i \in I_{D^{+}, C_{\text {source }}}\right\}$ in order to find disjoint sets $I_{D^{+}}^{\prime}$ and $J_{C_{s} \text { ource }}$ contained in $I_{D^{+}, C_{\text {source }}}$ such that $\left|I_{D^{+}}^{\prime}\right|=2 t 3,\left|J_{C_{s} \text { ource }}\right|=N+40$, and also for each $i \in I_{D^{+}}^{\prime}$ there is an $B_{i}^{\prime 1} \subseteq B_{i}^{1}$ of order 32 , and for each $j \in J_{C_{s} \text { ource }}$ there is a vertex $u_{j}^{+} \in B_{j}^{1}$. In addition we have all the edges going from $B_{i}^{\prime 1}$ to $u_{j}^{+}$for any $i \in I_{D^{+}}^{\prime}$ and $j \in J_{C_{s} \text { ource }}$.

Apply Lemma 4.16 to the family $\left\{A_{i}^{1}: i \in I_{D^{-}, C_{s i n k}}\right\}$ in order to find disjoint sets $I_{D^{-}}^{\prime}$ and $J_{C_{s} i n k}$ contained in $I_{D^{-}, C_{s i n k}}$ such that $\left|I_{D^{-}}^{\prime}\right|=2 t,\left|J_{C_{s} i n k}\right|=N+40$, and also for each $i \in I_{D^{-}}^{\prime}$ there is an $A_{i}^{\prime 1} \subseteq A_{i}^{1}$ of order 32, and for each $j \in J_{C_{s} i n k}$ there is a vertex $u_{j}^{-} \in A_{j}^{1}$. In addition we have all the edges going from $u_{j}^{-}$to $A_{i}^{\prime 1}$ for any $i \in I_{D^{-}}^{\prime}$ and $j \in J_{C_{s} \text { ink }}$.

Recall that for all $i$, vertices in $A_{i}^{1}$ have large in-degree and vertices in $B_{i}^{1}$ have large out-degree. In particular this means that $u_{j}^{-}$always has large in-degree and $u_{j}^{+}$always has large out-degree. Therefore since $\left|J_{C_{\text {source }}}\right|,\left|J_{C_{\text {sink }}}\right|=N+40 t$, we can apply Lemma $4.13 t$ times to $T$ with $\left\{x_{1}, \ldots, x_{N}\right\}=\left\{u_{j}^{+}: j \in J_{C_{s} \text { ource }}\right\}$ and $\left\{y_{1}, \ldots, y_{N}\right\}=\left\{u_{j}^{-}: j \in J_{C_{s} i n k}\right\}$ in order to find $t$ disjoint connectors $C_{1}, \ldots, C_{t}$ whose sources are in $\left\{u_{j}^{+}: j \in J_{C_{s} \text { ource }}\right\}$ and whose sinks are in $\left\{u_{j}^{-}: j \in J_{C_{s} i n k}\right\}$ (at each application of Lemma 4.13 we let $Y$ be $Z \backslash\left\{x_{1}, \ldots, x_{N}, y_{1}, \ldots, y_{N}\right\}$ together with the vertices of the previously constructed connectors. Condition (iv) ensures that $|Y| \leq|T| / 50$ as required by Lemma 4.13.) We let $S=\left(V\left(C_{1}\right) \cup \cdots \cup V\left(C_{t}\right)\right) \backslash$ $\left\{x_{1}, \ldots, x_{N}, y_{1}, \ldots, y_{N}\right\}$. Notice that we have $|S| \leq 40 t$ from the definition of "connector" and $S \cap Z=\emptyset$ from the construction of $C_{1}$, dots, $C_{t}$.

Notice that for two sets of numbers $A$ and $B$, either half of the numbers in $A$ are at least as large as half of the numbers of $B$, or half of the numbers of $B$ are at least as large as half of the numbers of $A$. Applying this with $A=\left\{\left|E_{i}^{-}\right|: i \in I_{D^{-}}^{\prime}\right\}$ and $B=\left\{\left|E_{i}^{+}\right|: i \in I_{D^{+}}^{\prime}\right\}$ gives us two subsets $I_{D^{+}} \subseteq I_{D^{+}}^{\prime}$ and $I_{D^{-}} \subseteq I_{D^{-}}^{\prime}$ with $\left|I_{D^{+}}\right|=t$ and $\left|I_{D^{-}}\right|=t$ such that we either have $\left|E_{i}^{-}\right| \geq\left|E_{j}^{+}\right|$for all $i \in I_{D^{-}}, j \in I_{D^{+}}$, or $\left|E_{i}^{-}\right| \leq\left|E_{j}^{+}\right|$for all $i \in I_{D^{-}}, j \in I_{D^{+}}$.

Now we have everything set up to define our $t$-linker.

- The indominators of $L$ are given by $D_{i}^{\prime-}=\left(A_{i}^{\prime 1}, A_{i}^{2}, A_{i}^{3}, A_{i}^{\prime 4}, E_{i}^{-}\right)$for $i \in I_{D^{-}}$. We reorder these indominators such that $\left|E_{1}^{-}\right| \geq\left|E_{2}^{-}\right| \geq \cdots \geq\left|E_{t}^{-}\right|$holds.

- The outdominators of $L$ are given by $D_{i}^{\prime+}=\left(B_{i}^{\prime 1}, B_{i}^{2}, B_{i}^{3}, B_{i}^{\prime 4}, E_{i}^{+}\right)$for $i \in I_{D^{+}}$. We reorder these outdominators such that $\left|E_{1}^{+}\right| \geq\left|E_{2}^{+}\right| \geq \cdots \geq\left|E_{t}^{+}\right|$holds.

- The connectors of $L$ are $C_{1}, \ldots, C_{t}$.

- The paths of $L$ are given by $Q_{j}^{\prime}$ for $j \in J_{Q}$.

It remains to check that we have constructed everything so that $L$ is a $t$-linker in $T$ with exceptional set $X \cup S$. Notice that $D_{i}^{\prime-}$ is an $(32,32,64)$-indominator with exceptional set $X$ for each $i$, since $D_{i}^{-}$was an $(32,64,64)$-indominator with exceptional set $X$, and we only removed vertices from the " $A^{1}$ " and " $A^{4}$ " sets 
of the indominator. For the same reason $D_{i}^{\prime+}$ is an $(32,32,64)$-outdominator with exceptional set $X$ for each $i$. Lemmas 4.10 and 4.11 together with (v) and $|S| \leq 40 t$ imply that $D_{i}^{\prime-}$ and $D_{i}^{\prime+}$ are $(32,32,32)$-dominators with exceptional set $X \cup S$. Conditions (L1) - (L3) are immediate from our construction. Condition (L4) follows from our choice of $I_{D^{-}}$and $I_{D^{+}}$. Finally all the edges in (L5) are present as a consequence of our applications of Lemmas 4.15 and 4.16 .

The fact that "for any $Y$ containing $X \cup S$ with $2|Y| \leq \delta^{+}(T), L$ is also a linker in $T$ with exceptional set $Y$ " follows immediately from Lemmas 4.10 and 4.11 , and the fact that $D_{i}^{-}$and $D_{i}^{+}$are $(32,32,64)$-dominators.

The following lemma allows us to find many linkers in a highly connected tournament.

Lemma 4.18. There is a constant $C_{0}=C_{0}(t)$ such that every $C_{0} k$-connected tournament contains $k$ vertex disjoint $t$-linkers with a common exceptional set $X$ of size $\leq C_{0} k$.

Proof. We first show that we can find many subdigraphs of $T$ satisfying the conditions of Lemma 4.17. Let $R_{0}=R_{0}(t)$ be the constant given by Lemma 4.17. We let $C_{1}=50\left(R_{0}+40 t\right)$ and $C_{0}=5^{103} C_{1}$. Let $T$ be a $C_{0} k$-connected tournament. Notice that this implies that $|T|, \delta^{+}(T), \delta^{-}(T) \geq C_{0} k$.

Claim 4.19. The tournament $T$ contains sets of vertices $X$ and $Z$, and $R_{0} k$ vertex disjoint digraphs $H_{1}, \ldots, H_{R_{0} k}$ satisfying parts (i) - (v) of Lemma 4.17 as well as $|X| \leq 5^{101} C_{1} k$ and $|Z| \leq|T| / 50-40 t k$.

Proof. By applying Lemma 4.8 repeatedly, we can choose $C_{1} k$ vertex disjoint $(32,64,128)$-indominators $D_{1}^{-}, \ldots, D_{C_{1} k}^{-}$of $T$, with a common exceptional set $X^{-}$of order at most $5^{100} C_{1} k$. Indeed to do this, we first apply Lemma 4.8 to $T$ with $m=32, M=64, p=256, L=5^{100}$, and $Y=\emptyset$ to find an $(32,64,256)$-indominator $D_{1}^{-}$with an exceptional set $X_{1}$ satisfing $\left|X_{1}\right| \leq 5^{100}$. Then for $i=2, \ldots, C_{1} k$, we apply Lemma 4.8 to $T$ with $m=32, M=64, p=256, L=5^{100}$, and $Y=V\left(D_{i-1}^{-}\right) \cup X_{i-1}$ in order to find a disjoint $(32,64,256)$ indominator $D_{i}^{-}$of $T$ with exceptional set $X_{i}$ satisfying $\left|X_{i}\right| \leq i 5^{100}$ and containing $Y$. Notice that we always have $|Y| \leq 5^{101} C_{1} k \leq|T| / 25-2^{2 m+2 M}$ and so are allowed to apply Lemma 4.8 in this way. Let $X^{-}=V\left(D_{C_{1} k}^{-}\right) \cup X_{C_{1} k}$ to get a set with $2\left|X^{-}\right| \leq \delta^{+}(T)$ and $X_{1} \subseteq X_{2} \subseteq \cdots \subseteq X_{C_{1} k} \subseteq X^{-}$. By Lemma 4.10, for each $i, D_{i}^{-}$is an $(32,64,128)$-indominator with exceptional set $X^{-}$.

By the same argument, using Lemma 4.9 we can choose $C_{1} k$ vertex disjoint $(32,64,64)$-outdominators $D_{1}^{+}, \ldots, D_{C_{1} k}^{+}$of $T$, with a common exceptional set $X$ of order at most $5^{101} C_{1} k$ containing $X^{-}$. By choosing $Y$ to contain $X^{-}$at each application of Lemma 4.8, we also ensure that $D_{i}^{+} \cap D_{j}^{-}=\emptyset$ for all $i$ and $j$. Since $2|X| \leq \delta^{+}(T)$ holds, Lemma 4.10 again implies that for each $i, D_{i}^{-}$is an $(32,64,64)$-indominator with exceptional set $X$.

Recall that Lemma 4.8 ensures that all the vertices in the $A^{1}$ set of the indominator it produces have large in-degree. Therefore, we have that all the vertices $A_{i}^{1}$ and $B_{i}^{1}$ have large in-degree and out-degree respectively (as will be required in part (ii) of Lemma 4.17).

Let $h_{1}^{-}, \ldots, h_{C_{1} k}^{-}$be the heads of the indominators $D_{1}^{-}, \ldots, D_{C_{1} k}^{-}$. Let $t_{1}^{+}, \ldots, t_{C_{1} k}^{+}$be the tails of the outdominators $D_{1}^{+}, \ldots, D_{C_{1} k}^{+}$. Let $T^{\prime}=\left(T \backslash \bigcup_{i=1}^{C_{1} k}\left(V\left(D_{i}^{-}\right) \cup V\left(D_{i}^{+}\right)\right)\right) \cup \bigcup_{i=1}^{C_{1} k}\left\{h_{i}^{-}, t_{i}^{+}\right\}$, i.e. $T^{\prime}$ is the subtournament of $T$ built by removing all the dominators we constructed, and then adding the heads and tails back in.

Since the dominators constructed above each have 192 vertices, $T^{\prime}$ is $\left(C_{0}-384 C_{1}\right) k$-connected. Since $C_{0}-384 C_{1} \geq C_{1}$, we can apply Menger's Theorem to find vertex disjoint paths $Q_{1}, \ldots, Q_{C_{1} k}$ such that $Q_{i}$ goes from $h_{i}^{-}$to $t_{\sigma(i)}^{+}$for some permutation $\sigma$ of $\left[C_{1} k\right]$. For each $i$, let $H_{i}=D_{i}^{-} \cup Q_{i} \cup D_{\sigma(i)}^{+}$.

Since the graphs $H_{i}$ are all vertex disjoint, the Pigeonhole Principle implies that there is a subset $I \subseteq\left[C_{1} k\right]$ of order $R_{0} k=C_{1} k / 50-40 t k$ such that $\left|\bigcup_{i \in I} H_{i}\right| \leq|T| / 50-40 t k$. Let $Z=\bigcup_{i \in I} V\left(H_{i}\right)$.

It is easy to check that the collection of graphs $\left\{H_{i}: i \in I\right\}$ together with the sets $X$ and $Z$ satisfy all the conditions of Lemma 4.17. Indeed (i) and (iii) hold from our construction of the dominators, paths, and sets $X$ and $Z$. Condition (ii) holds since Lemmas 4.8 and 4.9 ensured that all the vertices in $A_{i}^{1}$ and $B_{i}^{1}$ have large in-degrees and out-degrees respectively. Condition (iv) holds from our choice of $I$. Condition (v) holds since we have $\delta^{+}(T), \delta^{-}(T) \geq C_{0} k \geq 2(|X|+40 t)$.

Now partition $\left\{H_{1}, \ldots, H_{R_{0}+k}\right\}$ into $k$ collections $\mathcal{H}_{j}=\left\{H_{(j-1) R_{0}+1}, \ldots, H_{j R_{0}}\right\}$ for $j=1, \ldots, k$. Let $X_{1}=$ $X$ and $Z_{1}=Z$. Apply Lemma 4.17 to $\mathcal{H}_{1}$ with the sets $X_{1}$ and $Z_{1}$ to find a $t$-linker consisting of vertices in $\mathcal{H}_{1}$, plus a set of vertices $S_{1}$ of order at most $40 t$ disjoint from $Z_{1}$. Let $X_{2}=X_{1} \cup S_{1}$ and $Z_{2}=Z_{1} \cup S_{1}$. Then for each $i=2, \ldots, k$, apply Lemma 4.17 to $\mathcal{H}_{i}$ with the sets $X_{i}$ and $Z_{i}$ to find a $t$-linker $L_{i}$ consisting of vertices in $\mathcal{H}_{i}$, plus a set of vertices $S_{i}$ of order at most $40 t$ disjoint from $Z_{i}$ (at each step letting $X_{i}=X_{i-1} \cup S_{i-1}$ and $\left.Z_{i}=Z_{i-1} \cup S_{i-1}\right)$. This gives us a collection of $k$ disjoint linkers $L_{1}, \ldots, L_{k}$ with exceptional sets $X_{1}, \ldots, X_{k}$ respectively. Since $|X| \leq 5^{101} C_{1} k,\left|S_{i}\right| \leq 40 t$ and $X_{k}=X \cup S_{1} \cup \cdots \cup S_{k}$ we have $\left|X_{k}\right| \leq 5^{102} C_{1} k \leq C_{0} k$. The 
last part of Lemma 4.17 ensures that $L_{1}, \ldots, L_{k}$ are all $t$-linkers in $T$ with the common exceptional set $X_{k}$ as required.

\subsection{Properties of linkers}

In this section, we prove that families of linkers are linking families. First we will need to show that linkers have Hamiltonian paths between pairs of essential vertices. The following lemma shows that a 1-linker has a Hamiltonian path between certain prescribed pairs of vertices.

Lemma 4.20. Let $L$ be a 1-linker in a tournament $T$. Let $x$ and $y$ be two distinct vertices in $L$ such that $x$ is in the indominator of $L$ and $y$ is either in the outdominator of $L$ or a sink of the connector of $L$. Then $L$ contains a Hamiltonian path from $x$ to $y$.

Proof. Let $D^{-}=\left(A^{1}, A^{2}, A^{3}, A^{4}, E^{-}\right), D^{+}=\left(B^{1}, B^{2}, B^{3}, B^{4}, E^{+}\right)$, and $C$ be the indominator, outdominator, and connector of $L$ respectively, and $Q_{1}, Q_{2}, Q_{3}, Q_{4}, Q_{5}$ be the five paths of $L$.

First we'll consider the case when $y$ is in the outdominator of $L$. Let $P_{x}$ be a shortest path from $x$ to $A^{4}$. Let $P_{y}$ be a shortest path from $B^{4}$ to $y$. Let $P_{1}^{-}, P_{2}^{-}, P_{3}^{-}, P_{4}^{-}$be four paths, each from $A^{1}$ to $A^{4}$ such that $P_{x}$, $P_{1}^{-}, P_{2}^{-}, P_{3}^{-}, P_{4}^{-}$together partition $V\left(D^{-}\right)$(we can choose such disjoint paths using (D2) combined with the fact that $\left|A^{i}\right|=32$ for all $i$ ). Similarly, let $P_{1}^{+}, P_{2}^{+}, P_{3}^{+}, P_{4}^{+}$be four paths, each from $B^{4}$ to $B^{1}$ such that $P_{y}$, $P_{1}^{+}, P_{2}^{+}, P_{3}^{+}, P_{4}^{+}$together partition $V\left(D^{+}\right)$. From the definition of connector, we can partition $V(C)$ into four paths $R_{1}, \ldots, R_{4}$, each going from a source of $C$ to a sink. Now we have a Hamiltonian path from $x$ to $y$ formed by joining $P_{x}$ to $Q_{1}$ to $P_{1}^{+}$to $R_{1}$ to $P_{1}^{-}$to $Q_{2}$ to $P_{2}^{+}$to $R_{2}$ to $P_{2}^{-}$to $Q_{3}$ to $P_{3}^{+}$to $R_{3}$ to $P_{3}^{-}$to $Q_{4}$ to $P_{4}^{+}$to $R_{4}$ to $P_{4}^{-}$to $Q_{5}$ to $P_{y}$. Part (L5) of Definition 4.14 ensures that all the edges between the endpoints of these paths are oriented the correct way.

Now consider the case when $y$ is a sink of $C$. As in the previous case, let $P_{x}$ be a shortest path from $x$ to $A^{4}$, let $P_{1}^{-}, P_{2}^{-}, P_{3}^{-}, P_{4}^{-}$be four paths from $A^{1}$ to $A^{4}$ partitioning $V\left(D^{-}\right)$, let $P_{1}^{+}, P_{2}^{+}, P_{3}^{+}, P_{4}^{+}, P_{5}^{+}$be five paths, from $B^{4}$ to $B^{1}$ partitioning $V\left(D^{+}\right)$. From the definition of connector, we can partition $V(C)$ into five paths $R_{1}, \ldots, R_{5}$, each going from a source of $C$ to a sink. Since $y$ is a sink, one of these paths ends in $y$. Without loss of generality let this be $R_{5}$. Now we have a Hamiltonian path from $x$ to $y$ formed by joining $P_{x}$ to $Q_{1}$ to $P_{1}^{+}$to $R_{1}$ to $P_{1}^{-}$to $Q_{2}$ to $P_{2}^{+}$to $R_{2}$ to $P_{2}^{-}$to $Q_{3}$ to $P_{3}^{+}$to $R_{3}$ to $P_{3}^{-}$to $Q_{4}$ to $P_{4}^{+}$to $R_{4}$ to $P_{4}^{-}$to $Q_{5}$ to $P_{5}^{+}$to $R_{5}$.

The following lemma shows that a $t$-linker has a Hamiltonian path between prescribed pairs of vertices in its dominators.

Lemma 4.21. Let $L$ be a $t$-linker for any $t \geq 1$. Let $x$ be a vertex in one of the indominators of $L$ and $y$ a vertex in one of the outdominators of $L$. Then $L$ contains a Hamiltonian path from $x$ to $y$.

Proof. If $t=1$, then the lemma follows from Lemma 4.20, so suppose $t \geq 2$. We can partition $L$ into $t$-linkers $L_{1}, \ldots, L_{t}$ such that $L_{1}$ contains $x$ and $L_{t}$ contains $y$.

By Lemma 4.20, for we can find a Hamiltonian path $P_{1}$ in $L_{1}$ from $x$ to a sink of the connector of $L_{1}$. Similarly for $i=2, \ldots, t-1$, we can find a Hamiltonian path $P_{i}$ from a vertex in the $A^{1}$-set of $L_{i}$ to a sink of the connector of $L_{i}$. Finally, we can find a Hamiltonian path $P_{t}$ in $L_{t}$ from a vertex in the $A^{1}$-set of $L_{t}$ to $y$. Joining these together using the fact that there is an edge from any of the sinks of the connectors in a $t$-linker and the $A^{1}$-sets, gives the required Hamiltonian path in $L$.

The following lemma is the main property that linkers have. It says that under certain conditions on a tournament $T$, a 12-linker is a linking family of size $1 \mathrm{in} T$. This lemma will easily imply that a family of linkers is a linking family.

Lemma 4.22. Let $t$ and $K$ be integers satisfying $K / 5 \geq t \geq 12$. Let $T$ be a tournament with minimum in and out-degrees at least $80 K$. Suppose that we have a $t$-linker $L$ in $T$ with exceptional set $X$ such that $|X| \leq K$.

For $r \leq K$, suppose we have two vertices $x$ and $y \in V(T) \backslash V(L)$ and vertex disjoint paths $P_{1}, \ldots, P_{r}$ in $V(T) \backslash(V(L) \cup\{x, y\})$. Then there are vertex disjoint paths $P, P_{1}^{\prime}, \ldots, P_{r}^{\prime}$ such that

(i) $P$ is from $x$ to $y$.

(ii) $P_{j}^{\prime}$ has the same endpoints as $P_{j}$ for every $j$.

(iii) $V(P) \cup V\left(P_{1}^{\prime}\right) \cup \cdots \cup V\left(P_{r}^{\prime}\right)$ consists of $V\left(L_{i}\right) \cup V\left(P_{1}\right) \cup \cdots \cup V\left(P_{r}\right) \cup\{x, y\}$, plus at most 6 other vertices. 
Proof. We'll actually prove a slightly stronger statement about $t$-linkers for all $t \geq 1$. Suppose we have $t \geq 1$, and $K \geq 5 t, T, L$ and $X$ as in the statement of the lemma. Let the dominators, connectors, and paths of $L$ be labelled as in the Definition 4.14.

Notice that without loss of generality, we can assume that $\left|E_{t}^{-}\right| \geq\left|E_{1}^{+}\right|$occurs in (L4) for the linker $L$. Indeed otherwise, we could reverse all arcs in the tournament and exchange the roles of $x$ and $y$ in order to reduce to the case when $\left|E_{t}^{-}\right| \geq\left|E_{1}^{+}\right|$holds.

Let $x$ and $y$ be two vertices in $V(T) \backslash V(L)$. We will prove the lemma in several steps depending on where $x$ and $y$ lie.

Claim 4.23. Let $P_{1}, \ldots, P_{r}$ be vertex disjoint paths in $V(T) \backslash(V(L) \cup\{x, y\})$. Suppose that any of the following hold.

(a) $t \geq 1, r \leq K+9, m=0$, and $x \notin E_{i}^{-} \cup X$ and $y \notin E_{j}^{+} \cup X$ for some $i, j \leq t$.

(b) $t \geq 2, r \leq K+4, m=1$, and $x \notin X$ and $y \notin E_{j}^{+} \cup X$ for some $j \leq t$.

(c) $t \geq 4, r \leq K+2, m=2$, and $x \notin X$ and $y \notin X$.

(d) $t \geq 12, r \leq K$, and $m=6$.

Then there are vertex disjoint paths $P, P_{1}^{\prime}, \ldots, P_{r}^{\prime}$ such that

(i) $P$ is from $x$ to $y$.

(ii) $P_{j}^{\prime}$ has the same endpoints as $P_{j}$ for every $j$.

(iii) $V(P) \cup V\left(P_{1}^{\prime}\right) \cup \cdots \cup V\left(P_{r}^{\prime}\right)$ consists of $V\left(L_{i}\right) \cup V\left(P_{1}\right) \cup \cdots \cup V\left(P_{r}\right) \cup\{x, y\}$, plus at most $m$ other vertices.

The proof of parts (b) - (d) in the above claim are very similar. Therefore, to save space, we only write out the full details for the proof of part (b), and then explain how they should be modified for parts (c) and (d). For completeness we include more details of the proof of the above claim in the appendix.

The general strategy of the proofs of cases $(\mathrm{b})-(\mathrm{d})$ is as follows. We use the high out-degree of $x$ to find an out-neighbour $x_{1}$ of $x$ such that the pair of vertices " $x_{1}$ and $y$ " satisfy the condition of part (a) of Claim 4.23. If $x_{1}$ does not lie on any of the paths $P_{1}, \ldots, P_{r}$, then we are able to apply part (a) in order to prove the claim. If $x_{1}$ lies on some path $P_{i}$, then it will have neighbours $x_{2}$ and $y_{2}$ on this path. It turns out that $x_{1}$ can always be chosen such that the pair of vertices " $x_{2}$ and $y_{2}$ " can be joined together using part (a) of Claim 4.23. To prove the claim, we partition the linker $L$ into two sublinkers $L_{1}$ and $L_{2}$ with $L_{1}$ used to join $x_{1}$ to $y$, and $L_{2}$ used to "repair" the path $P_{i}$ by joining $x_{2}$ to $y_{2}$.

Proof of Claim 4.23. Let $Q_{1}, \ldots, Q_{5 t}$ be the paths of $L$. Let $U$ be the set of endpoints of the paths $P_{1}, \ldots, P_{r}$, and $W$ the set of endpoints of the paths $Q_{1}, \ldots, Q_{5 t}$. Notice that we have $|U|,|W| \leq 4 K$.

(a) Since $x \notin E_{i}^{-} \cup X$, there is some $x_{1} \in D_{i}^{-}$such that $x x_{1}$ is an edge. Similarly, since $y \notin E_{j}^{+} \cup X$, there is some $y_{1} \in D_{j}^{+}$such that $y_{1} y$ is an edge. Applying Lemma 4.21 to $L$ gives us a Hamiltonian path $R$ in $L$ from $x_{1}$ to $y_{1}$. Letting $P$ be the path formed by joining $x$ to $R$ to $y$ and $P_{i}^{\prime}=P_{i}$ for every $i$ proves the claim.

(b) If $x \notin E_{1}^{-}$then we are done by part (a). Therefore suppose that we have $x \in E_{1}^{-}$. Choose $\ell$ to be any integer between 1 and $t$ which is not $j$.

Since $x \in E_{1}^{-}$we have that $\left|N^{+}(x)\right| \geq 32\left|E_{1}^{-}\right|$. We also have $d^{+}(x) \geq 80 K$. Averaging these and using $|X| \leq K$ and $|U|,|W| \leq 4 K$ we obtain $\left|N^{+}(x)\right| \geq 4\left(\left|E_{1}^{-}\right|+|X|+|U|+|W|\right)$. Therefore there are at least $3\left(\left|E_{1}^{-}\right|+|X|+|U|+|W|\right)$ vertices in $\left|N^{+}(x)\right|$ outside of $E_{1}^{-} \cup X \cup U \cup W$. If one of these vertices, $x^{\prime}$, is not on any of the paths $P_{1}, \ldots, P_{r}, Q_{1}, \ldots, Q_{5 t}$ then we can let $P_{r+1}=\{x\}$, and apply part (a) to get a path $Q$ from $x^{\prime}$ to $y$ and then join $x$ to this path to prove the claim.

Therefore, we can suppose that all the vertices in $N^{+}(x) \backslash\left(E_{1}^{-} \cup X \cup U \cup W\right)$ are on the paths $P_{1}, \ldots, P_{r}, Q_{1}, \ldots, Q_{5 t}$. Since $\left|N^{+}(x) \backslash\left(E_{1}^{-} \cup X \cup U \cup W\right)\right| \geq 3\left(\left|E_{1}^{-}\right|+|X|+|U|+|W|\right)$ holds and $\left|E_{1}^{-}\right| \geq$ $\left|E_{\ell}^{-}\right|,\left|E_{\ell}^{+}\right|$, we can choose a vertex $x_{1}$ in $N^{+}(x) \backslash\left(E_{1}^{-} \cup X \cup U \cup W\right)$ such that $x_{1}$ is on a path $Q^{\prime} \in$ $\left\{Q_{1}, \ldots, Q_{5 t}, P_{1}, \ldots, P_{r}\right\}$, the predecessor of $x_{1}$ on $Q^{\prime}$ is not in $E_{\ell}^{-} \cup X$, and the successor of $x_{1}$ on $Q^{\prime}$ is not in $E_{\ell}^{+} \cup X$. We'll suppose for now that $Q^{\prime}$ is one of the paths $Q_{1}, \ldots, Q_{5 t}$. Without loss of generality $Q^{\prime}=Q_{5 t}$. Let $x_{2}$ be the predecessor of $x_{1}$ on $Q^{\prime}$ and $y_{2}$ the successor of $x_{1}$ on $Q^{\prime}$. Let $Q_{x}^{\prime}$ be the initial segment of $Q^{\prime}$ ending at the predecessor of $x_{2}$ and $Q_{y}^{\prime}$ the final segment of $Q^{\prime}$ starting at the successor of $y_{2}$. 
Let $L^{\prime}$ be a 1-linker contained in $L$ consisting of $D_{\ell}^{-}, D_{\ell}^{+}$, the connector $C_{\ell}$, and the paths $Q_{1}, \ldots, Q_{5}$. Now let $T^{\prime}$ be the subtournament of $T$ formed by removing the essential vertices of $L$, and adding the essential vertices of $L^{\prime}$ back in. It is easy to check that $L^{\prime}$ is still a 1-linker in $T^{\prime}$ (using the fact that all the vertices we removed from $T$ were in the exceptional set $X$ ). Apply part (a) to $T^{\prime}$ with the vertices $x_{2}, y_{2}, 1$-linker $L^{\prime}$, and paths $\left\{Q_{x}^{\prime}, Q_{y}^{\prime}\right\} \cup\left\{P_{1}, \ldots, P_{r}, Q_{6}, \ldots, Q_{5 t-1}\right\}$ as well as three one-vertex paths $\{x\},\{y\}$, and $\left\{x_{1}\right\}$. This gives us disjoint paths $Q_{x}^{\prime \prime}, Q_{y}^{\prime \prime}, P_{1}^{\prime}, \ldots, P_{r}^{\prime}, Q_{6}^{\prime}, \ldots, Q_{5 t-1}^{\prime}$ with the same endpoints as the previous paths, and a new path $R$ starting at $x_{2}$ and ending at $y_{2}$. In addition all these paths avoid $x, y$, and $x_{1}$, and the union of their vertices is $V\left(L^{\prime}\right) \cup V\left(P_{1}\right) \cup \cdots \cup V\left(P_{r}\right) \cup V\left(Q_{1}\right) \cup \cdots \cup V\left(Q_{5 t-1}\right) \cup V\left(Q_{x}^{\prime}\right) \cup V\left(Q_{y}^{\prime}\right) \cup\left\{x_{2}, y_{2}\right\}$. Let $Q_{5 t}^{\prime}$ be the path formed by joining $Q_{x}^{\prime \prime}$ to $R$ to $Q_{y}^{\prime \prime}$.

Let $L^{\prime \prime}$ be the $(t-1)$-linker formed from $L$ by removing $L^{\prime}$ and replacing $Q_{i}$ by $Q_{i}^{\prime}$ for each $i$. Now we can apply part (a) in $T$ with the linker $L^{\prime \prime}$, vertices $x_{1}$ and $y$, and paths $P_{1}^{\prime}, \ldots, P_{r}^{\prime},\{x\}$. This gives us paths $P_{1}^{\prime \prime}, \ldots, P_{r}^{\prime \prime}$ as well as a path $P$ from $x_{1}$ to $y$. Joining $x$ to $P$ gives the required collection of paths.

The case when $Q^{\prime}$ was one of the paths $P_{1}, \ldots, P_{r}$ is proved identically.

(c) If $y \notin E_{1}^{+}$then we are done by part (b). Therefore suppose that we have $y \in E_{1}^{+}$.

Since $y \in E_{1}^{+}$we have that $\left|N^{-}(y)\right| \geq 32\left|E_{1}^{+}\right|$. As before, there are at least $3\left(\left|E_{1}^{+}\right|+|X|+|U|+|W|\right)$ vertices in $\left|N^{-}(y)\right|$ outside of $E_{1}^{+} \cup X \cup U \cup W$. If one of these vertices, $y^{\prime}$, is not on any of the paths $P_{1}, \ldots, P_{r}, Q_{1}, \ldots, Q_{5 t}$ then we can let $P_{r+1}=\{y\}$, and apply part (b) to get a path $Q$ from $x$ to $y^{\prime}$ and then join this path to $y$ to prove the claim.

Therefore, we can suppose that all the vertices in $N^{-}(y) \backslash\left(E_{1}^{+} \cup X \cup U \cup W\right)$ are on the paths $P_{1}, \ldots, P_{r}, Q_{1}, \ldots, Q_{5 t}$. Since $\left|N^{-}(y) \backslash\left(E_{1}^{+} \cup X \cup U \cup W\right)\right| \geq 3\left(\left|E_{1}^{+}\right|+|X|+|U|+|W|\right)$ and $\left|E_{1}^{+}\right| \geq\left|E_{2}^{+}\right|$ hold, we can choose a vertex $y_{1}$ in $N^{-}(y) \backslash\left(E_{1}^{+} \cup X \cup U \cup W\right)$ such that $y_{1}$ is on a path $Q^{\prime} \in$ $\left\{Q_{1}, \ldots, Q_{r}, P_{1}, \ldots, P_{5 t}\right\}$ for some $i$, and the neighbours of $y_{1}$ on this path are in neither $E_{2}^{+}$nor $X$. Let $x_{2}$ be the predecessor of $y_{1}$ on $Q^{\prime}$ and $y_{2}$ the successor of $y_{1}$ on $Q^{\prime}$.

The rest of the proof is nearly identical to the proof of part (b), so we only sketch it. The full proof of this case is in the appendix. We choose a 2-linker $L^{\prime}$ contained in $L$ such that $D_{2}^{+}$is one of the outdominators of $L^{\prime}$ and $L^{\prime}$ doesn't contain the path $Q^{\prime}$. We remove the essential vertices of the linker $L$ from $T$ and add $L^{\prime}$ back in to obtain a tournament $T^{\prime}$. Apply part (b) to $T^{\prime}$ with the linker $L^{\prime}$ in order to join $x_{2}$ to $y_{2}$ by a path. Then let $L^{\prime \prime}$ be the $(t-2)$-linker in $T$ formed by removing $L^{\prime}$ from $L$. Applying part (b) to $T$ with the linker $L^{\prime \prime}$ allows us to join $x$ to $y_{1}$ (and then to $y$ ) as required.

(d) Notice that since $T$ has minimum out-degree $\geq 80 K, x$ has at least $7|X \cup U \cup W|$ out-neighbours outside of $X \cup U \cup W$. Similarly, since $T$ has minimum in-degree $\geq 80 K, y$ has at least $7|X \cup U \cup W|$ inneighbours outside of $X \cup U \cup W$. Suppose for now that all such neighbours of $x$ and $y$ lie on the paths $P_{1}, \ldots, P_{r}, Q_{1}, \ldots, Q_{5 t}$. Then we can choose an out-neighbour $x_{1}$ of $x$, and a distinct in-neighbour $y_{1}$ of $y$, such that $x_{1}$ and $y_{1}$ are outside of $X \cup U \cup W$. In addition, since $d^{+}(x) \geq 7|X \cup U \cup W|, x_{1}$ can be chosen to lie on some path with predecessor $x_{2}$ and successor $y_{2}$ with $x_{2}, y_{2} \notin X$. Similarly $y_{1}$ can be chosen to lie on some path with predecessor $x_{3}$ and successor $y_{3}$, such that $x_{3}, y_{3} \notin X$.

Similarly how we did in cases (b) and (c), we can partition the linker $L$ into three sublinkers, and then apply part (c) three times in order to join $x_{2}$ to $y_{2}$ then $x_{3}$ to $y_{3}$, and finally $x_{1}$ to $y_{1}$.

The cases when $x$ and/or $y$ have neighbours outside of $X \cup U \cup W$ and the paths $P_{1}, \ldots, P_{r}, Q_{1}, \ldots, Q_{5 t}$ are very similar. The only difference is that since we won't need to join the pairs vertices " $x_{2}, y_{2}$ " and/or " $x_{3}, y_{3}$ " we would be able to find the required $x-y$ path using just one or two applications of part (c).

The lemma follows since it is exactly part (d) of the claim.

So far we have only considered linkers in tournaments. In Theorem 2.5, we will actually need linkers in digraphs. For a digraph $D$ and a subgraph $L \subseteq D$, we say that $L$ is a $t$-linker in $D$ with exceptional set $X \subseteq V(D)$ if there is some tournament on the vertices $V(D)$ containing $D$ in which $L$ is a $t$-linker in $T$ with exceptional set $X$. We'll need the following version of Lemma 4.22 for digraphs.

Lemma 4.24. Let $t$ and $K$ be integers satisfying $K / 5 \geq t \geq 12$. Let $D$ be a digraph with minimum degree at least $|D|-K$ and minimum in and out-degrees at least $81 K$. Suppose that we have a $t$-linker $L$ in $D$ with exceptional set $X$ such that $|X| \leq K$.

For $r \leq K$, suppose we have two vertices $x$ and $y \in V(D) \backslash V(L)$ and vertex disjoint paths $P_{1}, \ldots, P_{r}$ in $V(D) \backslash(V(L) \cup\{x, y\})$. Then there are vertex disjoint paths $P, P_{1}^{\prime}, \ldots, P_{r}^{\prime}$ such that

(i) $P$ is from $x$ to $y$. 
(ii) $P_{j}^{\prime}$ has the same endpoints as $P_{j}$ for every $j$.

(iii) $V(P) \cup V\left(P_{1}^{\prime}\right) \cup \cdots \cup V\left(P_{r}^{\prime}\right)$ consists of $V\left(L_{i}\right) \cup V\left(P_{1}\right) \cup \cdots \cup V\left(P_{r}\right) \cup\{x, y\}$, plus at most 6 other vertices.

The above lemma has an identical proof to Lemma 4.22. The only difference is that vertices in $D$ may have slightly smaller degree than they did in $T$, but since that all vertices in $D$ have minimum in and out-degree $81 K$, this is not significant in any of the inequalities in the proof of Lemma 4.22 . Given the similarity between Lemmas 4.22 and 4.24 , we omit the proof of Lemma 4.24 .

Now we use Lemma 4.24 to prove that a family of linkers is a linking family.

Lemma 4.25. Let $t$ and $K$ be integers satisfying $K / 5 \geq t \geq 12$. Let $D$ be a digraph with minimum degree at least $|D|-K / 2$ and minimum in and out-degrees at least $82 K$.

Suppose that for $k$ satisfying $296 t k \leq K / 2$, we have a family of vertex disjoint $t$-linkers $L_{1}, \ldots, L_{k}$ in $D$ with common exceptional set $X$ such that $|X| \leq K$. Then $\left\{L_{1}, \ldots, L_{k}\right\}$ is a linking family in $D$.

Proof. Fix $t, K$ and $D$ as in the statement of the lemma. The proof is by induction on $k$. Suppose that the statement is false. Let $k_{0}$ be the minimal value of $k$ for which it is false.

Let $L_{1}, \ldots, L_{k_{0}}$ be a family of $k_{0}$ vertex disjoint $t$-linkers with common exceptional set $X$ as in the lemma. Let $\mathcal{Q}_{1}, \ldots, \mathcal{Q}_{k_{0}}$ be the families of paths of these linkers. Let $x, y$ be two vertices, $m \leq 100 k_{0}$, and $P_{1}, \ldots, P_{m}$ paths as in the definition of "linking family".

Let $D^{\prime}$ be $D$ with the essential vertices of $L_{1}, \ldots, L_{k_{0}-1}$ removed. Notice that $D^{\prime}$ has minimum degree at least $|D|-K / 2-296 t k_{0} \geq|D|-K$ and minimum in and out-degrees at least $82 K-296 t k_{0} \geq 81 K$. Also notice that the total number of paths in $\left\{P_{1}, \ldots, P_{m}\right\} \cup Q_{i} \cup \cdots \cup Q_{k_{0}-1}$ is at most $100 k_{0}+5 t\left(k_{0}-1\right) \leq K$. Therefore we can apply Lemma 4.24 in $D^{\prime}$ with the linker $L_{k_{0}}$, vertices $x$ and $y$, and paths $P_{1}, \ldots, P_{m}$, plus all the paths in $\mathcal{Q}_{1}, \ldots, \mathcal{Q}_{k_{0}-1}$. This gives us an $x-y$ path $P$, and new paths $P_{1}^{\prime}, \ldots, P_{m}^{\prime}$, and families of paths $\mathcal{Q}_{1}^{\prime}, \ldots, \mathcal{Q}_{k_{0}}^{\prime}$. Since for each $i$, the paths in $\mathcal{Q}_{i}^{\prime}$ have the same endpoints as those in $\mathcal{Q}_{i}$ we can define a new $t$-linker $L_{i}^{\prime}$ formed by replacing the paths in $L_{i}$ with those in $\mathcal{Q}_{i}^{\prime}$.

Now we claim that the paths $P, P_{1}^{\prime}, \ldots, P_{m}^{\prime}$, and digraphs $L_{1}^{\prime}, \ldots, L_{k_{0}}^{\prime}$ satisfy (i) - (iv) in the definition of "linking family". Conditions (i) - (iii) are immediate from our application of Lemma 4.24. If $k_{0}>1$, then (iv) holds by minimality of $k_{0}$, and if $k_{0}=1$ then (iv) holds vacuously. This shows that $\left\{L_{1}, \ldots, L_{k_{0}}\right\}$ is a linking family in $D$, which contradicts our assumption that the lemma was false for $k=k_{0}$.

\subsection{Proof of Theorem 2.5}

Putting together Lemmas 4.18 and 4.25 it is easy to prove Theorem 2.5.

Proof of Theorem 2.5. Let $C_{0}=C_{0}(12)$ be the constant from Lemma 4.18. Let $\Delta_{1}$ be the maximum degree of a 12-linker. Set $C_{1}=8300 \Delta_{1} C_{0}$.

Let $T$ be any $C_{1} k$ connected tournament. By Lemma 4.18, we can find $k$ vertex disjoint 12 -linkers $L_{1}, \ldots, L_{k}$ in $T$ with a common exceptional set $X$ satisfying $|X| \leq C_{0} k$.

Let $K=100 \Delta_{1} C_{0} k$. Notice that this ensures that we have $|X| \leq K$ and $296 \cdot 12 \cdot k \leq K / 2$. Let $D$ be a subdigraph of $T$ satisfying $\delta(D) \geq|T|-50 \Delta_{1} C_{0} k=|T|-K / 2$. Notice that since $T$ is $C_{1}$-connected, it must satisfy $\delta^{-}(T), \delta^{+}(T) \geq C_{1} k$ and so $\delta^{-}(D), \delta^{+}(D) \geq\left(C_{1}-50 \Delta_{1} C_{0}\right) k \geq 82 K$. Now we can apply Lemma 4.25 in order to conclude that for any subfamily $\mathcal{L} \subseteq\left\{L_{1}, \ldots, L_{k}\right\}$ is a linking family in $D \cup \mathcal{L}$.

\section{Concluding remarks}

We close with some remarks and open problems.

- For clarity of presentation, we made no attempt to optimize the constant $C$ in Theorem 1.4. In future work it might be interesting to investigate how small this constant can be made, or to see whether exact bounds on the connectivity can be obtained for small $k$. For $k=2$, Thomassen conjectured that every strongly 3-connected tournament contains 2 edge-disjoint Hamiltonian cycles [13].

- There are a several open problems in this area. One is the following conjecture of Kühn, Osthus, and Townsend.

Conjecture 5.1 (Kühn, Osthus, and Townsend, [9]). There is a constant $C$ such that the vertices of every strongly $C t k$-connected tournament can be partitioned into $t$ strongly $k$-connected subtournaments. 
The existence of a function $f(t, k)$ for which every strongly $f(t, k)$-connected tournament can be partitioned into $t$ strongly $k$-connected subtournaments was a conjecture of Thomassen. This conjecture was solved by Kühn, Osthus, and Townsend using a version of Theorem 2.1. The only $k$ for which a linear bound is known is $k=1$, where $f(t, 1)=t$ was proved by Chen, Gould, and Li [4].

Another is a conjecture of Song [11], which says that for any natural numbers $n_{1}, \ldots, n_{k}$ satisfying $\sum_{i=1}^{k} n_{i}=n$, every sufficiently large $k$-connected tournament $T$ on $n$ vertices can be partitioned into cycles $C_{1}, \ldots, C_{k}$ such that $\left|C_{i}\right|=n_{i}$. Kühn, Osthus, and Townsend showed that this is true with the condition that " $T$ is $k$-connected" is replaced by " $f(k)$-connected" for a suitable function $f(k)$. As an intermediate step to Song's conjecture it would be interesting to show that $f(k)$ can be linear.

Problem 5.2. Show that there is a constant $M$, such that for any natural numbers $n_{1}, \ldots, n_{k}$ satisfying $\sum_{i=1}^{k} n_{i}=n$, the vertices of every strongly $M k$-connected tournament $T$ on $n$ vertices can be partitioned into cycles $C_{1}, \ldots, C_{k}$ such that $\left|C_{i}\right|=n_{i}$.

Finally, as a tool for studying the above conjectures it would be interesting to know how small the bound on the connectivity in Theorem 2.1 can be.

\section{Acknowledgement}

The author would like to thank John Lapinskas for finding a mistake in an earlier draft of this paper and suggesting a nice fix. The author would also like to thank a careful reviewer for suggesting numerous improvements to this paper.

\section{References}

[1] J. Bang-Jensen. Problems and conjectures concerning connectivity, paths, trees and cycles in tournamentlike digraphs. Discrete Math., 309:5655-5667, 2009.

[2] J. Bang-Jensen and G. Gutin. Digraphs: Theory, Algorithms and Applications. Springer, 2008.

[3] P. Camion. Chemins et circuits hamiltoniens des graphes complets. C. R. Acad. Sci. Paris, 249:2151-2152, 1959.

[4] G. Chen, R. Gould, and H. Li. Partitioning vertices of a tournament into independent cycles. J. Combin. Theory B, 83:213-220, 2001.

[5] T. Gallai and A. Milgram. Verallgemeinerung eines graphentheoretischen satzes von rédei. Acta Sc. Math., 21:181-186, 1960.

[6] D. Kühn, J. Kim, and D. Osthus. Bipartitions of highly connected tournaments. arXiv:1411.1533, 2014.

[7] D. Kühn, J. Lapinskas, D. Osthus, and V. Patel. Proof of a conjecture of Thomassen on Hamilton cycles in highly connected tournaments. Proc. London Math. Soc., 109:733-762, 2014.

[8] D. Kühn and D. Osthus. A survey on Hamilton cycles in directed graphs. European J. Combin., 33:750-766, 2012 .

[9] D. Kühn, D. Osthus, and T. Townsend. Proof of a tournament partition conjecture and an application to 1-factors with prescribed cycle lengths. Combinatorica, 35:1-19, 2015.

[10] A. Pokrovskiy. Highly linked tournaments. J. Combin. Theory B, to appear, 2015.

[11] Z. M. Song. Complementary cycles of all lengths in tournaments. J. Combin. Theory B, 57:18-25, 1993.

[12] C. Thomassen. Hamiltonian-connected tournaments. J. Combin. Theory B, 28:142-163, 1980.

[13] C. Thomassen. Edge-disjoint Hamiltonian paths and cycles in tournaments. Proc. London Math. Soc., 45:151-168, 1982.

[14] C. Thomassen. Connectivity in tournaments. In Graph Theory and Combinatorics, a volume in honour of Paul Erdős (B. Bollobás, ed.), pages 305-313. Academic Press, London, 1984. 